\title{
Time-Reproductive solutions for a bioconvective flow
}

\author{
B. Climent-Ezquerra, L. Friz ${ }^{\dagger}$ and M. A. Rojas-Medar ${ }^{\dagger}$
}

\begin{abstract}
We consider the existence and uniqueness of periodic solutions for the generalized bioconvective flow, which is a well known model to describe the convection caused by the concentration of upward swimming microorganism in a fluid.
\end{abstract}

Keywords: Bioconvective flow, periodic solution, Navier-Stokes type equations.

AMS Subjects Classification 35Q35, 76D03, 76Z99

\section{Introduction}

We show results of existence of periodic solution to the equations of flow Bioconvective Generalized, which describe the motion of a viscous fluid and incompressible in which there exists culture of microorganisms. Understand by bioconvective, convection derived from difference in concentration of microorganisms in culture, due to the fact that these microorganisms have a tendency to swim the vertical direction (we are considering the case of a domain where the flow is submitted to the action of forces gravitational).

The equations discussed in this work are:

$$
\left\{\begin{array}{l}
\frac{\partial \boldsymbol{u}}{\partial t}-2 \operatorname{div}(\mu(c) D(\boldsymbol{u}))+\boldsymbol{u} \cdot \nabla \boldsymbol{u}+\nabla p=-g(1+\rho c) \chi+\boldsymbol{f} \\
\operatorname{div} \boldsymbol{u}=0, \\
\frac{\partial c}{\partial t}-\theta \Delta c+\boldsymbol{u} \cdot \nabla c+U \frac{\partial c}{\partial x_{3}}=0, \quad \text { on } \quad(0, T) \times \Omega .
\end{array}\right.
$$

Here the following notations are used

- $\Omega \subset \mathbb{R}^{3}$ is a bounded domain and represents the region of flow of fluid. It denotes $\partial \Omega$ the boundary of $\Omega$.

${ }^{*}$ Dpto. de Ecuaciones Diferenciales y Análisis Numérico, Universidad de Sevilla. Apdo 1160, 41080 Sevilla, España. E-mail: bcliment@us.es.

${ }^{\dagger}$ Grupo de Matemática Aplicada, Dpto. de Ciencias Básicas, Facultad de Ciencias, Universidad del Bío-Bío, Campus Fernando May, Casilla 447, Chillán, Chile. E-mails: marko@ueubiobio.cl, lfriz@roble.fdo-may.ubiobio.cl 
- $\boldsymbol{u}(x, t)$ denotes the fluid velocity at a point $x=\left(x_{1}, x_{2}, x_{3}\right) \in \Omega$, and instant $t \in[0, T]$, where $0<T \leq+\infty$.

- $p(x, t)$ is the hydrostatic pressure at the point $x$ and instant $t$.

- $c(x, t)$ represents the concentration of microorganisms at a point $x \in \Omega$ and instant $t$.

- $\mu(\cdot)>0$ is the viscosity of fluid.

- $\theta$ is a constant that indicates the rate of diffusion of microorganisms.

- $g$ is the intensity of the acceleration of gravity (assumed constant).

- $\boldsymbol{f}$ represents an external force given. We will suppose that $\boldsymbol{f}$ is divided in two parts, $\tilde{\boldsymbol{f}}$ which does not depend on $t$ and $\hat{\boldsymbol{f}}$ that depends on $t$.

- $\chi$ is a unitary vector in the vertical direction, i.e. $\chi=(0,0,1)^{t}$. That is, coordinate system is placed so that the gravitational forces acting on vertical.

- $U$ denotes the average velocity of swimming of the microorganisms in the vertical direction.

- $\rho$ is a positive constant, given by $\rho=\frac{\rho_{0}}{\rho_{m}}-1$, where $\rho_{0}$ and $\rho_{m}$ are the density of one organism and the culture fluid density, respectively.

In the above equations, $\nabla, \Delta$ and div represent the gradient, Laplacian and divergence operator, respectively; $\boldsymbol{u} \cdot \nabla \boldsymbol{u}$ indicates the convection operator, whose component $i$-th in cartesian coordinates is given by $(\boldsymbol{u} \cdot \nabla \boldsymbol{u})_{i}=\sum_{j=1}^{3} u_{j} \frac{\partial u_{i}}{\partial x_{j}}$. The operator $D(\boldsymbol{u})=\frac{1}{2}\left(\nabla \boldsymbol{u}+(\nabla \boldsymbol{u})^{t}\right)$ represent the stress-tensor.

In what follows, the spaces labeled in bold face represent the cartesian product of the space with itself three times.

In order to simplify the notation, we set $c=(g \rho)^{-1} m$ and $p=q-g x_{3}, \quad \mu(c)=\nu(m)$, then (1) is rewritten as

$$
\left\{\begin{array}{l}
\frac{\partial \boldsymbol{u}}{\partial t}-2 \operatorname{div}(\nu(m) D(\boldsymbol{u}))+\boldsymbol{u} \cdot \nabla \boldsymbol{u}+\nabla q=-m \chi+\mathbf{f}, \\
\operatorname{div} \boldsymbol{u}=0, \\
\frac{\partial m}{\partial t}-\theta \Delta m+\boldsymbol{u} \cdot \nabla m+U \frac{\partial m}{\partial x_{3}}=0, \quad \text { in } \quad(0, T) \times \Omega .
\end{array}\right.
$$

The classical bioconvective equations correspond to the particular case of (1) with the viscosity $\mu$ constant (during of course of this work call this special case of classical bioconvective equations). Levandowsky and others [15] and Moribe [12] discuss in their work, the biological and physical phenomena that give rise to such equations. They also have some qualitative 
descriptions based on intuitive arguments. Kan-On, Narukawa and Teramoto [9] do a mathematical analysis of the classical bioconvective equations, obtaining results of existence of solutions both of the stationary and evolution problems.

We note that in this paper we are interested in the generalized case in which the concentration of microorganisms affects the viscosity of the fluid. This problem, with initial conditions, was studied in the papers [2], [3], both for stationary and evolution problem, proving weak and strong solutions. The uniqueness question also was studied. Now, we study the problem with periodic conditions and for this we use techniques similar to those in [4], [5], [6].

The system of equations (2) will be considered together with the following boundary conditions

$$
\left\{\begin{array}{l}
\boldsymbol{u}=0 \quad \text { on } \quad(0, T) \times S, \\
\boldsymbol{u} \cdot \mathbf{n}=0 \quad \text { on } \quad(0, T) \times \Gamma, \\
\nu(m)[D(\boldsymbol{u}) \mathbf{n}-\mathbf{n} \cdot(D(\boldsymbol{u}) \mathbf{n}) \mathbf{n}]=0 \quad \text { on } \quad(0, T) \times \Gamma, \\
\theta \frac{\partial m}{\partial \mathbf{n}}-U m n_{3}=0 \quad \text { on } \quad(0, T) \times \partial \Omega .
\end{array}\right.
$$

and periodic conditions:

$$
\boldsymbol{u}(0)=\boldsymbol{u}(T), \quad m(0)=m(T), \quad \text { in } \quad \Omega .
$$

Here, $\partial \Omega=S \cup \Gamma$, is composed of a rigid boundary $S$ and a plane free surface $\Gamma$, both orthogonal at the points of intersection. We are going to consider $S$ and $\Gamma$ known and time independent. The vector $\mathbf{n}(x)=\left(n_{1}(x), n_{2}(x), n_{3}(x)\right)$ is the exterior unitary normal vector at the point $x \in$ $\partial \Omega$ and $\frac{\partial}{\partial \mathbf{n}}$ is the normal derivative on $\partial \Omega$. The first and second equation of (3) on $\Gamma$ are the so called slip conditions for fluid velocity and they are often used to model conditions about free boundary in fluids (see Joseph [8]). In particular, the third one corresponds to attribute to the tangential component of the stress vector null value (free boundary condition). Solonnikov and Scadilov [19], Mulone and Salemi [18] analyze in their works the classical Navier-Stokes equations with this type of the boundary conditions for domains with free boundary.

By other hand, the third equation of (1) is associated to the equation of conservation $\frac{d}{d t} c+$ $\operatorname{div} J=0, x \in \Omega, t>0$, for $\frac{d}{d t}=\frac{\partial}{\partial t}+\boldsymbol{u} \cdot \nabla$ and $J$ the flow of microorganisms given by $J=-\theta \nabla c+U c \chi$. The fourth equation of (9) establishes the condition of null flow at each point $x \in \partial \Omega$. Kan-On and others [9] use this boundary conditions for the concentration of microorganisms in culture.

In this work, as in Kan-On, Narukawa and Teramoto [9], we will assume that average velocity of swimming $U$ is constant. In summary, in this work we will be interested in discuss results of existence and uniqueness of periodic solutions of the system (2)-(4) when $U$ is constant. 


\section{Preliminaries and auxiliary results}

In this Section we fix the notation that we will use in the paper and also we recall some results. Let $\Omega \subseteq \mathbb{R}^{3}$ be a bounded domain with boundary of class $C^{3}$. Let $H^{m}(\Omega)$ be the usual Sobolev spaces on $\Omega$ with norm $\|\cdot\|_{m}(m$ real $),(\cdot, \cdot)$ denote the usual inner product in $L^{2}(\Omega)$ and $|\cdot|$ denote the $L^{2}$-norm on $\Omega$. By $H_{0}^{1}(\Omega)$ we denote the completion of $C_{0}^{\infty}(\Omega)$ under the norm $\|\cdot\|_{1}$, the $L^{p}$-norm on $\Omega$ is denoted by $|\cdot|_{p}, 1 \leq p \leq \infty$. If $B$ is a Banach space, we denote by $L^{q}(0, T: B)$ the Banach space of the $B$-valued functions defined in the interval $(0, T)$ that are $L^{q}$-integrable in the sense of Bochner.

Let $\partial \Omega=S \cup \Gamma$ be with $S$ and $\Gamma$ disjoint. Assume that $S$ and $\Gamma$ are sufficiently smooth, we define

$$
\dot{H}(\Omega)=\left\{\boldsymbol{u} \in \boldsymbol{C}_{0}^{\infty}(\bar{\Omega}) ;\left.\boldsymbol{u}\right|_{S}=0,\left.\boldsymbol{u} \cdot \mathbf{n}\right|_{\Gamma}=0\right\},
$$

$H(\Omega)$ clousure of $\dot{H}(\Omega)$ with respect to norm \|\|$_{H(\Omega)}$.

Here $\|\cdot\|_{H(\Omega)}$ denote the norm given by

$$
\|\boldsymbol{u}\|_{H(\Omega)}=\left[\int_{\Omega} \nabla \boldsymbol{u}: \nabla \boldsymbol{u} d x\right]^{1 / 2}=[(\nabla \boldsymbol{u}, \nabla \boldsymbol{u})]^{1 / 2}=|\nabla \boldsymbol{u}| .
$$

Also, $Y$ will be denote the closed subspace of $L^{2}(\Omega)$ consisting of orthogonal functions to the constants, that is

and

$$
Y=\left\{f \in L^{2}(\Omega) ; \int_{\Omega} f(x) d x=0\right\}
$$

$$
B=H^{1}(\Omega) \cap Y .
$$

The following result can be found in [11]

Lemma 1 (Poincaré-Friedrichs inequality). Let $\Omega$ be a bounded domain of $\mathbb{R}^{3}$ with boundary $\partial \Omega$, of class $C^{1}$. Let $\Sigma \subseteq \partial \Omega$ be a part of the boundary on which the normal has three independent directions, then there exists a positive constant $C_{\Omega}$ depending only on the domain $\Omega$ such that

$$
|\boldsymbol{u}| \leq C_{\Omega}|\nabla \boldsymbol{u}|, \quad \text { for all } \quad \boldsymbol{u} \in \boldsymbol{H}^{1}(\Omega),\left.\boldsymbol{u} \cdot \mathbf{n}\right|_{\Sigma}=0 .
$$

The following lemma is given in [19], pp.191.

Lemma 2 (Korn inequality). Let $\Omega$ be a bounded domain of $\mathbb{R}^{3}$ with boundary $\partial \Omega$ of class $C^{2}$. Then there exists a positive constant $\bar{c}$, such that:

$$
\|\boldsymbol{u}\|_{H(\Omega)}=|\nabla \boldsymbol{u}| \leq \bar{c}|D(\boldsymbol{u})|, \quad \forall \boldsymbol{u} \in H(\Omega)
$$

Lemma 3 Under the hypothesis of above lemma, there exists $\gamma$ depending of $\Omega$ and $\partial \Omega$, such that

$$
|\boldsymbol{u}|^{2} \leq \gamma|D(\boldsymbol{u})|^{2}, \quad \forall \boldsymbol{u} \in H(\Omega)
$$


Thus, in $H(\Omega)$ the norms $|\nabla \boldsymbol{u}|$ and $|D(\boldsymbol{u})|$ are equivalent.

We denote by

$$
\boldsymbol{C}_{0, \sigma}^{\infty}(\Omega)=\left\{\boldsymbol{f} \in \boldsymbol{C}_{0}^{\infty}(\Omega): \operatorname{div} \boldsymbol{f}=0\right\}
$$

and

$$
X(\Omega)=\text { clousure of } \boldsymbol{C}_{0, \sigma}^{\infty}(\Omega) \text { in } \boldsymbol{L}^{2}(\Omega) .
$$

It is well known, see for instance, Temam [20]

$$
\boldsymbol{L}^{2}(\Omega)=X(\Omega) \oplus G(\Omega),
$$

where

$$
G(\Omega)=\left\{\boldsymbol{w} \in \boldsymbol{L}^{2}(\Omega), \boldsymbol{w}=\nabla q, q \in H^{1}(\Omega)\right\} .
$$

Also, we consider the following spaces

$$
\dot{J}(\Omega)=\{\boldsymbol{u} \in \dot{H}(\Omega), \operatorname{div} \boldsymbol{u}=0\}
$$

and $J_{0}(\Omega)$ is the closure of $\dot{J}(\Omega)$ in the norm (5).

The following lemma is proved in [13].

Lemma 4 Under the as hypotheses of Lemma 2, there exists a positive constant $C_{\Omega}$, such that

$$
|\phi| \leq C_{\Omega}|\nabla \phi|, \quad \forall \phi \in H^{1}(\Omega) \cap Y .
$$

It is easy deduce, from the convection-diffusion equation for $\eta$, the equality $\frac{d}{d t} \int_{\Omega} \eta(x, t)=0$. Then, we can fix $\eta$ such that $\int_{\Omega} \eta=0$. Therefore, let us consider the following spaces

$$
H_{N}^{k}=\left\{\eta \in H^{k} ; \theta \frac{\partial \eta}{\partial \mathbf{n}}-U \eta n_{3}=0 \text { on } \partial \Omega, \int_{\Omega} \eta=0\right\}
$$

where $k=2,3$. Hence, $H_{N}^{k}$ is a closed subspace of $H^{k}$. Consequently $|\Delta \eta|_{2}$ is equivalent to $\|\eta\|_{2}$ in $H_{N}^{2}$ and $|\nabla \Delta \eta|_{2}$ is equivalent to $\|\eta\|_{3}$ in $H_{N}^{3}$ (See [7], [14]).

\section{Lemma 5 (Some interpolation inequalities)}

$$
\begin{gathered}
|v|_{6} \leq C\|v\|_{1}, \quad|v|_{3} \leq|v|_{2}^{1 / 2}\|v\|_{1}^{1 / 2} \quad \forall v \in H^{1}, \\
|v|_{\infty} \leq C\|v\|_{1}^{1 / 2}\|v\|_{2}^{1 / 2} \quad \forall v \in H^{2} . \\
|v|_{L^{2}(\partial \Omega)} \leq C|v|_{2}^{1 / 2-\delta}\|v\|_{1}^{1 / 2+\delta} \quad \forall v \in H^{1+\delta}, \delta \in \mathbb{R} .
\end{gathered}
$$

See [1] or [10]. 
Lemma 6 The Stokes operator $A=-P \Delta$ with domain $D(A)=\left\{\boldsymbol{u} \in J_{0}(\Omega) \cap \boldsymbol{H}^{2}(\Omega) ; D(\boldsymbol{u}) \mathbf{n}-\right.$ $\left.\left.\mathbf{n} \cdot(D(\boldsymbol{u}) \mathbf{n}) \mathbf{n}\right|_{\Gamma}=0\right\}$ is positive definite, self-adjoint and its inverse operator $A^{-1}$ is compact, thus its spectrum is discrete, positive, and the finite multiplicity, the eigenvalues $\left\{\alpha_{i}\right\}, i \in \mathbb{N}$ converge to $+\infty$, the eigenfunctions $\left\{\mathbf{w}^{i}\right\}$ are orthogonal and complete in $X$ and in $J_{0}, J_{0} \cap \boldsymbol{H}^{2}(\Omega)$ and satisfy

$$
D\left(\mathbf{w}^{i}\right) \cdot \mathbf{n}-\left.\mathbf{n} \cdot D\left(\mathbf{w}^{i}\right) \cdot \mathbf{n n}\right|_{\Gamma}=0 .
$$

See [18].

By other hand, we denote by $\bar{P}$ the orthogonal projection of $L^{2}(\Omega) \rightarrow Y$. The following lemma was proved by Kan-On [9], pp. 150-152.

Lemma 7 The operator $A_{1}=-\bar{P} \Delta$ with domain

$$
D\left(A_{1}\right)=\left\{\varphi \in Y \cap H^{2}(\Omega) ; \theta \frac{\partial \varphi}{\partial \boldsymbol{n}}-U n_{3} \varphi=0 \text { on } \partial \Omega\right\},
$$

is positive definite, self-adjoint and its inverse operator is compact. Therefore, $-\bar{P} \Delta$ has a spectrum discrete, positive, with finite multiplicity. The eigenvalues $\left\{\beta_{i}\right\}, i \in \mathbb{N}$ converge to $+\infty$, the eigenfunctions $\left\{\phi^{i}\right\}$ are orthogonal and complete in $Y$.

Also, $P_{n}$ will denote the projection $P_{n}: \boldsymbol{L}^{2}(\Omega) \rightarrow \boldsymbol{V}_{n}$, where $\boldsymbol{V}_{n}=\left\langle\boldsymbol{w}^{1}, \boldsymbol{w}^{2}, \ldots, \boldsymbol{w}^{n}\right\rangle$ and $\widetilde{P}_{n}: L^{2}(\Omega) \rightarrow W_{n}$, with $W_{n}=\left\langle\phi^{1}, \phi^{2}, \ldots, \phi^{n}\right\rangle$. We use in the future the following result proved in $[17]$.

Lemma 8 Let $\boldsymbol{u} \in \boldsymbol{V} \cap \boldsymbol{H}^{2}$ and consider the Helmholtz decomposition of $-\Delta \boldsymbol{u}$, i.e. $-\Delta \boldsymbol{u}=$ $A \boldsymbol{u}+\nabla q$, where $q \in H^{1}$ is taken such that $\int_{\Omega} q d x=0$ and $A$ is the Stokes operator. Then,

$$
\|q\|_{1} \leq C|A \boldsymbol{u}|_{2}
$$

Moreover, for every $\varepsilon>0$ there exists a positive constant $C_{\varepsilon}$ (independent of $\boldsymbol{u}$ ) such that

$$
|q|_{2} \leq C_{\varepsilon}|\nabla \boldsymbol{u}|_{2}+\varepsilon|A \boldsymbol{u}|_{2} .
$$

The following Green formula will be fundamental, the proof is similar to that given by Solonnikov [19].

Lemma 9 Let $\boldsymbol{u}, \boldsymbol{v} \in J_{0}$ and $q \in C^{1}$. then,

$$
\int_{\Omega}[-2 \operatorname{div}(\nu(m) D(\boldsymbol{u}))+\nabla q] \boldsymbol{v} d x=2(\nu(m) D(\boldsymbol{u}), D(\boldsymbol{v}))
$$

We consider the stationary problem associated to (2),

$$
\left\{\begin{array}{l}
-2 \operatorname{div}(\nu(m) D(\boldsymbol{u}))+\boldsymbol{u} \cdot \nabla \boldsymbol{u}+\nabla q=-m \chi+\tilde{\boldsymbol{f}} \\
\operatorname{div} \boldsymbol{u}=0, \\
-\theta \Delta m+\boldsymbol{u} \cdot \nabla m+U \frac{\partial m}{\partial x_{3}}=0, \quad \text { in } \Omega .
\end{array}\right.
$$


together with the following boundary and initial conditions:

$$
\left\{\begin{array}{l}
\boldsymbol{u}=0 \quad \text { on } S, \\
\boldsymbol{u} \cdot \mathbf{n}=0 \quad \text { on } \quad \Gamma, \\
\nu(m)[D(\boldsymbol{u}) \mathbf{n}-\mathbf{n} \cdot(D(\boldsymbol{u}) \mathbf{n}) \mathbf{n}]=0 \quad \text { on } \quad \Gamma, \\
\theta \frac{\partial m}{\partial \mathbf{n}}-U m n_{3}=0 \quad \text { on } \quad \partial \Omega .
\end{array}\right.
$$

Let us recall that $\tilde{f}$ is the stationary part of the external force $\boldsymbol{f}$. The following result is been proved (see [2], [3]):

Theorem 10 Let $\boldsymbol{f} \in X(\Omega)$. Assume that $\nu$ is a function of class $C^{1}$ satisfying $0<\nu_{\min } \leq$ $\nu(s)<\nu_{\max }$ and $U$ small enough. Then, there exists a strong solution, $\left(\boldsymbol{u}_{\alpha}, q_{\alpha}, m_{\alpha}\right)$, of (6), (7) verifying

$$
\left(\boldsymbol{u}_{\alpha}, m_{\alpha}\right) \in\left(J_{0}(\Omega) \cap \boldsymbol{H}^{2}(\Omega)\right) \times\left(Y \cap \boldsymbol{H}^{2}(\Omega)\right) .
$$

Now, if we consider the changes of variables

$$
\boldsymbol{v}=\boldsymbol{u}-\boldsymbol{u}_{\alpha}, \quad \eta=m-m_{\alpha},
$$

then (2) is rewritten as follows

$$
\left\{\begin{array}{l}
\frac{\partial \boldsymbol{v}}{\partial t}-2 \operatorname{div}\left(\nu\left(\eta+m_{\alpha}\right) D(\boldsymbol{v})\right)-2 \operatorname{div}\left(\nu\left(\eta+m_{\alpha}\right) D\left(\boldsymbol{u}_{\alpha}\right)\right) \\
+2 \operatorname{div}\left(\nu\left(m_{\alpha}\right) D\left(\boldsymbol{u}_{\alpha}\right)\right)+\boldsymbol{v} \cdot \nabla \boldsymbol{v}+\boldsymbol{v} \cdot \nabla \boldsymbol{u}_{\alpha}+\boldsymbol{u}_{\alpha} \cdot \nabla \boldsymbol{v}+\nabla\left(q-q_{\alpha}\right)=-\eta \cdot \chi+\hat{\boldsymbol{f}} \\
\operatorname{div} \boldsymbol{v}=0 \\
\frac{\partial \eta}{\partial t}-\theta \Delta \eta+\boldsymbol{v} \cdot \nabla \eta+\boldsymbol{v} \cdot \nabla m_{\alpha}+\boldsymbol{u}_{\alpha} \cdot \nabla \eta+U \frac{\partial \eta}{\partial x_{3}}=0, \operatorname{in}(0, T) \times \Omega .
\end{array}\right.
$$

with the initial and boundary conditions

$$
\left\{\begin{array}{l}
\boldsymbol{v}=0 \text { on }(0, T) \times S \\
\boldsymbol{v} \cdot \mathbf{n}=0 \text { on }(0, T) \times \Gamma \\
\nu\left(\eta+m_{\alpha}\right)\left[D\left(\boldsymbol{v}+\boldsymbol{u}_{\alpha}\right) \mathbf{n}-\mathbf{n} \cdot\left(D\left(\boldsymbol{v}+\boldsymbol{u}_{\alpha}\right) \mathbf{n}\right) \mathbf{n}\right]=0, \text { on }(0, T) \times \Gamma \\
\theta \frac{\partial \eta}{\partial \mathbf{n}}-U \eta n_{3}=0 \text { on }(0, T) \times \partial \Omega .
\end{array}\right.
$$

\section{Existence of periodic solution}

\subsection{Existence of the reproductive solution}

In this section we seek reproductive solution, namely, a pair $(\boldsymbol{v}, \eta)$ solution of (8),(9) satisfying:

$$
\boldsymbol{v}(0)=\boldsymbol{v}(T), \quad \eta(0)=\eta(T) .
$$


Theorem 11 Let $\boldsymbol{f} \in L^{2}(0, T ; X(\Omega)), \boldsymbol{v}_{0} \in J_{0}(\Omega)$ and $\eta_{0} \in H^{2}(\Omega) \cap Y$. Assume that $\nu$ is a function of class $C^{1}$ satisfying $0<\nu_{\min } \leq \nu(s)<\nu_{\max }$ and $\sup \left|\nu^{\prime}(s)\right|<\nu_{\max }^{\prime}<\infty$ in $\mathbb{R}$. We consider $\left(\boldsymbol{u}_{\alpha}, q_{\alpha}, m_{\alpha}\right)$ a stationary solution obtained in Theorem 10. Then, for $\left|D\left(\boldsymbol{u}_{\alpha}\right)\right|,\left|\nabla m_{\alpha}\right|$ small enough there exists reproductive solution for the problem (8)

Proof. At first, we need to obtain an a priori estimate for the solution of the problem (8). Given $\left(\boldsymbol{w}^{j}\right)_{1}^{\infty}$ and $\left(\phi^{j}\right)_{1}^{\infty}$ eigenfunctions of $A$ and $A_{1}$ respectively, we consider Galerkin approximations of the form

$$
\boldsymbol{v}^{n}(t, x)=\sum_{j=1}^{n} \bar{c}_{n, j}(t) \boldsymbol{w}^{j}(x), \quad \eta^{n}(t, x)=\sum_{\ell=j}^{n} \bar{d}_{n, j}(t) \phi^{j}(x),
$$

solutions of the variational formulation of $(8)$

$$
\begin{aligned}
& \left(\frac{\partial \boldsymbol{v}^{n}}{\partial t}, \boldsymbol{w}^{j}\right)+2\left(\nu\left(\eta^{n}+m_{\alpha}\right) D\left(\boldsymbol{v}^{n}\right), D\left(\boldsymbol{w}^{j}\right)+2\left(\nu\left(\eta^{n}+m_{\alpha}\right) D\left(\boldsymbol{u}_{\alpha}\right), D\left(\boldsymbol{w}^{j}\right)\right)\right. \\
& -2\left(\nu\left(m_{\alpha}\right) D\left(\boldsymbol{u}_{\alpha}\right), D\left(\boldsymbol{w}^{j}\right)\right)+\left(\boldsymbol{v}^{n} \cdot \nabla \boldsymbol{v}^{n}, \boldsymbol{w}^{j}\right)+\left(\boldsymbol{v}^{n} \cdot \nabla \boldsymbol{u}_{\alpha}, \boldsymbol{w}^{j}\right) \\
& +\left(\boldsymbol{u}_{\alpha} \cdot \nabla \boldsymbol{v}^{n}, \boldsymbol{w}^{j}\right)+\left(\left(\eta^{n}\right) \chi, \boldsymbol{w}^{j}\right)=\left(\hat{\boldsymbol{f}}, \boldsymbol{w}^{j}\right) \\
& \left(\partial_{t} \eta^{n}, \phi^{j}\right)+\theta\left(\nabla \eta^{n}, \nabla \phi^{j}\right)+\left(\boldsymbol{v}^{n} \cdot \nabla \eta^{n}, \phi^{j}\right)+\left(\boldsymbol{v}^{n} \cdot \nabla m_{\alpha}, \phi^{j}\right)+\left(\boldsymbol{u}_{\alpha} \cdot \nabla \eta^{n}, \phi^{j}\right) \\
& -U\left(\eta^{n}, \frac{\partial}{\partial x_{3}} \phi^{j}\right)=0 \\
& \boldsymbol{v}^{n}(0)=P_{n} \boldsymbol{v}_{0}, \quad \eta^{n}(0)=\widetilde{P}_{n} \eta_{0} .
\end{aligned}
$$

By multiplying (10) by $c_{n, j}$, summing with respect to $j$, we obtain

$$
\begin{aligned}
& \frac{1}{2} \frac{\partial}{\partial t}\left|\boldsymbol{v}^{n}\right|^{2}+2\left(\nu\left(\eta^{n}+m_{\alpha}\right) D\left(\boldsymbol{v}^{n}\right), D\left(\boldsymbol{v}^{n}\right)\right)+2\left(\nu\left(\eta^{n}+m_{\alpha}\right) D\left(\boldsymbol{u}_{\alpha}\right), D\left(\boldsymbol{v}^{n}\right)\right)- \\
& \left.2\left(\nu\left(m_{\alpha}\right) D\left(\boldsymbol{u}_{\alpha}\right), D\left(\boldsymbol{v}^{n}\right)\right)\right)+\left(\boldsymbol{v}^{n} \cdot \nabla \boldsymbol{u}_{\alpha}, \boldsymbol{v}^{n}\right)=-\left(\eta^{n} \cdot \chi, \boldsymbol{v}^{n}\right)+\left(\hat{\boldsymbol{f}}, \boldsymbol{v}^{n}\right) .
\end{aligned}
$$

In a similar manner, we have

$$
\frac{1}{2} \frac{d}{d t}\left|\eta^{n}\right|^{2}+\theta\left|\nabla \eta^{n}\right|^{2}+\left(\boldsymbol{v}^{n} \cdot \nabla m_{\alpha}, \eta^{n}\right)-U\left(\eta^{n}, \frac{\partial \eta^{n}}{\partial x_{3}}\right)=0
$$

By Holder inequalities

$$
\begin{aligned}
\frac{1}{2} \frac{\partial}{\partial t}\left|\boldsymbol{v}^{n}\right|^{2}+2 \nu_{\min }\left|D\left(\boldsymbol{v}^{n}\right)\right|^{2} & \leq 4 \nu_{\max }\left|D\left(\boldsymbol{u}_{\alpha}\right)\right|\left|D\left(\boldsymbol{v}^{n}\right)\right|+\left|D\left(\boldsymbol{u}_{\alpha}\right)\right|\left|\boldsymbol{v}^{n}\right|_{4}^{2}+\left|\eta^{n}\right|\left|\boldsymbol{v}^{n}\right|+|\hat{\boldsymbol{f}}|\left|\boldsymbol{v}^{n}\right| \\
\frac{1}{2} \frac{d}{d t}\left|\eta^{n}\right|^{2}+\theta\left|\nabla \eta^{n}\right|^{2} & \leq\left|\boldsymbol{v}^{n}\right|_{4}\left|\nabla m_{\alpha}\right|\left|\eta^{n}\right|_{4}+U\left|\eta^{n}\right|\left|\nabla \eta^{n}\right|
\end{aligned}
$$


We put $\left|D\left(\boldsymbol{u}_{\alpha}\right)\right|,\left|\nabla m_{\alpha}\right|$ small enough so that $\left(\left|D\left(\boldsymbol{u}_{\alpha}\right)\right|^{2}+\left|\nabla m_{\alpha}\right|^{2}\right)<\frac{\nu_{\min }}{C}$, for a given constant $C$. Then, we obtain

$$
\frac{d}{d t}\left(\left|\boldsymbol{v}^{n}\right|^{2}+\left|\eta^{n}\right|^{2}\right)+\nu_{\min }\left|D\left(\boldsymbol{v}^{n}\right)\right|^{2}+\theta\left|\nabla \eta^{n}\right|^{2} \leq C\left(\left|D\left(\boldsymbol{u}_{\alpha}\right)\right|^{2}+\left|\eta^{n}\right|^{2}+|\hat{\boldsymbol{f}}|^{2}\right) .
$$

Integrating in $[0, t]$ and by applying Gronwall inequality we obtain that

$$
\left|\boldsymbol{v}^{n}\right|^{2}+\left|\eta^{n}\right|^{2}+\nu_{\min } \int_{0}^{t}\left|D\left(\boldsymbol{v}^{n}\right)\right|^{2}+\theta \int_{0}^{t}\left|\nabla \eta^{n}\right|^{2}
$$

is bounded by a continuous, bounded function, which not depend on $n$ for every $t$. Then, from (14) we obtain

$$
\frac{d}{d t}\left(\left|\boldsymbol{v}^{n}\right|^{2}+\left|\eta^{n}\right|^{2}\right)+C_{3}\left(\left|\boldsymbol{v}^{n}\right|^{2}+\left|\eta^{n}\right|^{2}\right) \leq H(t)
$$

where $H$ is a function in $\boldsymbol{L}^{1}(0, T)$, independent of $n$. Therefore, by multiplying by $e^{C_{3} t}$ and integrating in $[0, t]$ we have,

$$
e^{C_{3} t}\left(\left|\boldsymbol{v}^{n}(t)\right|^{2}+\left|\eta^{n}(t)\right|^{2}\right) \leq\left(\left|\boldsymbol{v}^{n}(0)\right|^{2}+\left|\eta^{n}(0)\right|^{2}\right)+\int_{0}^{t} H(s) d s
$$

Given $\left(\boldsymbol{v}_{0 n}, \eta_{0 n}\right) \in \boldsymbol{V}_{n} \times W_{n}$, we define the application $L^{n}:[0, T] \rightarrow \mathbb{R}^{n} \times \mathbb{R}^{n}$ as $L^{n}(t)=$ $\left(\bar{c}_{n, 1}(t), \ldots, \bar{c}_{n, n}(t), \bar{d}_{n, 1}(t), \ldots, \bar{d}_{n, n}(t)\right)$, where $\bar{c}_{n, i}(t), \bar{d}_{n, i}(t), i=1, \ldots, n$, are the coefficients of the expansion of $\boldsymbol{v}^{n}(t)$ and $\eta^{n}(t)$ in $V^{n}$ and $W^{n}$ respectively, being $\left(\boldsymbol{v}^{n}, \eta^{n}\right)$ the unique aproximate solution of system (10)-(11) with initial condition $\left(\boldsymbol{v}_{0 n}, \eta_{0 n}\right)$. Notice that $\left\|L^{n}(t)\right\|_{\mathbb{R}^{n} \times \mathbb{R}^{n}}^{2}=$ $\left|\boldsymbol{v}^{n}(t)\right|^{2}+\left|\eta^{n}(t)\right|$.

Given $L_{0}^{n} \in \mathbb{R}^{n} \times \mathbb{R}^{n}$, define $\Upsilon^{n}\left(L_{0}^{n}\right)=L^{n}(T)$, where $L^{n}(t)$ are the coefficients of the Galerkin solution $\left(\boldsymbol{v}^{n}, \eta^{n}\right)$ with initial value with coefficients $L_{0}^{n}$. We want to prove that $\Upsilon^{n}$ has a fixed point. For this, we will use the Leray-Schauder Theorem. Then, we need to show that for all $\lambda \in[0,1]$, the solutions $L_{0}^{n}(\lambda)$ of the equation

$$
L_{0}^{n}(\lambda)=\lambda \Upsilon^{n}\left(L_{0}^{n}(\lambda)\right)
$$

are bounded independently of $\lambda$. Since $L_{0}^{n}(0)=0$, it is sufficient to consider $\lambda \in(0,1]$. This is equivalent to $\Upsilon^{n}\left(L_{0}^{n}(\lambda)\right)=\frac{1}{\lambda} L_{0}^{n}(\lambda)$. Moreover, by definition of $\Upsilon^{n}$ and (17) we obtain

$$
e^{C_{3} t}\left\|\frac{1}{\lambda} L_{0}^{n}(\lambda)\right\|_{\mathbb{R}^{n} \times \mathbb{R}^{n}} \leq\left\|L_{0}^{n}(\lambda)\right\|_{\mathbb{R}^{n} \times \mathbb{R}^{n}}+\int_{0}^{t} H(s) d s
$$

which implies

$$
\left\|L_{0}^{n}(\lambda)\right\|_{\mathbb{R}^{n} \times \mathbb{R}^{n}} \leq \frac{\int_{0}^{t} H(s) d s}{e^{C_{3} t}-1}
$$

for each $\lambda \in(0,1]$. This bound is independent of $\lambda \in[0,1]$ and $n$. Consequently, LeraySchauder Theorem implies the existence of at least one fixed point of $\Upsilon^{n}$, that is, the existence of reproductive Galerkin solution. Finally, by standard arguments of compacity we conclude the existence of reproductive solution. 


\subsection{Existence of the periodic solution}

We seek periodic solution, namely, a pair $(\boldsymbol{v}, \eta)$ solution of (8) satisfying:

$$
\boldsymbol{v}(t)=\boldsymbol{v}(t+T), \quad \eta(t)=\eta(t+T) \quad \forall t \in \mathbb{R} .
$$

Definition 12 It will be said that $(\boldsymbol{u}, p, \eta)$ is a regular solution of $(2)-(4)$ in $(0, T)$, if

$$
\begin{gathered}
\boldsymbol{u} \in L^{2}\left(\boldsymbol{H}^{2}\right) \cap L^{\infty}\left(\boldsymbol{H}^{1}\right), \quad \partial_{t} \boldsymbol{u} \in L^{2}\left(\boldsymbol{L}^{2}\right) \quad \text { and } \quad p \in L^{2}\left(H^{1}\right), \\
\eta \in L^{2}\left(H_{N}^{3}\right) \cap L^{\infty}\left(H_{N}^{2}\right) \quad \text { and } \quad \partial_{t} \eta \in L^{2}\left(H_{N}^{1}\right),
\end{gathered}
$$

satisfying (2) a.e. in $(0, T) \times \Omega$, boundary conditions (9) and time reproductivity conditions (4) in the sense of spaces $\boldsymbol{V}$ and $H_{N}^{2}$ respectively.

Theorem 13 Let $\boldsymbol{f} \in L^{2}(0, \infty ; X(\Omega))$ be a t-periodic function with period T. Assume that $\nu$ is a function of class $C^{1}$ satisfying $0<\nu_{\min } \leq \nu(s)<\nu_{\max }$ and $\sup \left|\nu^{\prime}(s)\right|<\nu_{\max }^{\prime}<\infty$ in $\mathbb{R}$. We consider $\left(\boldsymbol{u}_{\alpha}, q_{\alpha}, m_{\alpha}\right)$ a stationary solution obtained in Theorem 10. Then, for $\nu_{\min }$ big enough and $\left\|v_{0}\right\|_{1},\left\|\eta_{0}\right\|_{2}$ and $\left|\partial_{t} \eta_{0}\right|_{1}$ small enough, there exists periodic solution for the problem (8), (9).

To prove this theorem, we first need to demonstrate some differential inequalities, then, using these inequalities, we shall prove that in the interval $[0, T]$ there is a reproductive solution. Finally, thanks to the regularity of solutions, we will deduce the existence of periodic solution.

\section{Differential inequalities in regular norms}

In the sequel, $\varepsilon$ will denote some constant sufficiently small. By $C$ we will denote different constants, independent on data and $\varepsilon$ and $\nu_{\min }$. In order to make calculations more simple, we suppose that $\hat{f}=0$.

Lemma 14 For each $\varepsilon>0$ sufficiently small, there exists constants $C=C(\varepsilon)>0$, such that

$$
\begin{aligned}
\frac{d}{d t} \int_{\Omega}( & \left.\nu\left(\eta^{n}+m_{\alpha}+1\right)\right)\left|D\left(\boldsymbol{v}^{n}\right)\right|^{2}+\frac{\nu_{\min }}{2}\left\|\boldsymbol{v}^{n}\right\|_{2}^{2}+\frac{1}{2}\left|\partial_{t} \boldsymbol{v}^{n}\right|_{2}^{2} \leq \varepsilon\left\|\boldsymbol{v}^{n}\right\|_{2}^{2}\left(\left\|\eta^{n}\right\|_{2}+\left\|m_{\alpha}\right\|_{2}\right) \\
& +\frac{C}{\nu_{\min }}\left\|\boldsymbol{v}^{n}\right\|_{2}^{2}\left(\left\|\boldsymbol{v}^{n}\right\|_{1}^{2}+\left\|\eta^{n}\right\|_{2}^{2}+\left|\partial_{t} \eta^{n}\right|_{2}^{2}+\left\|\boldsymbol{u}_{\alpha}\right\|_{2}^{2}+\left\|m_{\alpha}\right\|_{2}^{2}\right) \\
& +\frac{C}{\nu_{\min }}\left(\left\|\eta^{n}\right\|_{2}^{2}\left(\left\|\boldsymbol{u}_{\alpha}\right\|_{2}^{2}+1\right)+\left\|\boldsymbol{u}_{\alpha}\right\|_{2}^{2}\left(\left\|m_{\alpha}\right\|_{2}^{2}+1\right)\right) \\
& +C\left[\left\|\boldsymbol{v}^{n}\right\|_{1}^{2}\left\|\boldsymbol{u}_{\alpha}\right\|_{2}^{2}+\left\|\eta^{n}\right\|_{2}^{2}\left(\left\|\boldsymbol{u}_{\alpha}\right\|_{2}^{2}+1\right)+\left\|\boldsymbol{u}_{\alpha}\right\|_{2}^{2}\left(\left\|m_{\alpha}\right\|_{2}^{2}+1\right)\right] .
\end{aligned}
$$


Proof. First, taking $\boldsymbol{v}=A \boldsymbol{v}^{n}$ as test function in the $\boldsymbol{v}^{n}$-system (10) ( $A$ is the Stokes operator mentioned in Lemma 6) one has

$$
\begin{aligned}
\left(\partial_{t} \boldsymbol{v}^{n}, A \boldsymbol{v}^{n}\right) & -2\left(\nabla \cdot\left(\nu\left(\eta^{n}+m_{\alpha}\right) D\left(\boldsymbol{v}^{n}\right)\right), A \boldsymbol{v}^{n}\right)-2\left(\nabla \cdot\left(\nu\left(\eta^{n}+m_{\alpha}\right) D\left(\boldsymbol{u}_{\alpha}\right)\right), A \boldsymbol{v}^{n}\right) \\
& +2\left(\nabla \cdot\left(\nu\left(m_{\alpha}\right) D\left(\boldsymbol{u}_{\alpha}\right)\right), A \boldsymbol{v}^{n}\right)+\left(\left(\boldsymbol{v}^{n} \cdot \nabla\right) \boldsymbol{v}^{n}, A \boldsymbol{v}^{n}\right) \\
& +\left(\left(\boldsymbol{v}^{n} \cdot \nabla\right) \boldsymbol{u}_{\alpha}, A \boldsymbol{v}^{n}\right)+\left(\left(\boldsymbol{u}_{\alpha} \cdot \nabla\right) \boldsymbol{v}^{n}, A \boldsymbol{v}^{n}\right)=-\left(\eta^{n} \chi, A \boldsymbol{v}^{n}\right) .
\end{aligned}
$$

We can write the first term as

$$
\left(\partial_{t} \boldsymbol{v}^{n}, A \boldsymbol{v}^{n}\right)=-\left(\partial_{t} \boldsymbol{v}^{n}, 2 \nabla \cdot\left(D\left(\boldsymbol{u}^{n}\right)\right)\right)=\frac{d}{d t}\left|D\left(\boldsymbol{v}^{n}\right)\right|^{2}-2 \int_{\Gamma} \partial_{t} \boldsymbol{v}^{n}\left(D\left(\boldsymbol{u}^{n}\right) \mathbf{n}\right) .
$$

By splitting $\partial_{t} \boldsymbol{v}^{n}$ and $D\left(\boldsymbol{u}^{n}\right) \mathbf{n}$ in their tangential and normal components:

$\left.\left.D\left(\boldsymbol{v}^{n}\right) \mathbf{n}=\left(D\left(\boldsymbol{v}^{n}\right) \mathbf{n}\right) \cdot \mathbf{n}\right) \mathbf{n}+\left(D\left(\boldsymbol{v}^{n}\right) \mathbf{n}-\left(D\left(\boldsymbol{v}^{n}\right) \mathbf{n}\right) \cdot \mathbf{n}\right) \mathbf{n}\right), \quad \partial_{t} \boldsymbol{v}=\left(\partial_{t} \boldsymbol{v} \cdot \mathbf{n}\right) \mathbf{n}+\left(\partial_{t} \boldsymbol{v}-\left(\partial_{t} \boldsymbol{v} \cdot \mathbf{n}\right) \mathbf{n}\right)$, taking into account that $\partial_{t} \boldsymbol{v} \cdot \mathbf{n}=0$ and $\left.D\left(\boldsymbol{v}^{n}\right) \mathbf{n}-\left(D\left(\boldsymbol{v}^{n}\right) \mathbf{n}\right) \cdot \mathbf{n}\right) \mathbf{n}=0$ on $\partial \Omega$, we obtain that

$$
\left(\partial_{t} \boldsymbol{v}^{n}, A \boldsymbol{v}^{n}\right)=\frac{d}{d t}\left|D\left(\boldsymbol{v}^{n}\right)\right|^{2} .
$$

On the other hand, by using the identity $2 \nabla \cdot(\nu(m) D(\boldsymbol{u}))=\nu(m) \Delta \boldsymbol{u}+2 \nu^{\prime}(m) \nabla m D(\boldsymbol{u})$ and the Helmholtz decomposition $\Delta \boldsymbol{v}^{n}=-A \boldsymbol{v}^{n}+\nabla \bar{q}_{m}$, the second term of (20) is equal to

$$
\begin{aligned}
& -2\left(\nabla \cdot\left(\nu\left(\eta^{n}+m_{\alpha}\right) D\left(\boldsymbol{v}^{n}\right)\right), A \boldsymbol{v}^{n}\right)=\left(\nu\left(\eta^{n}+m_{\alpha}\right) A \boldsymbol{v}^{n}, A \boldsymbol{v}^{n}\right) \\
& \quad-\left(\nu\left(\eta^{n}+m_{\alpha}\right) \nabla \bar{q}_{m}, A \boldsymbol{v}^{n}\right)-2\left(\nu^{\prime}\left(\eta^{n}+m_{\alpha}\right) \nabla\left(\eta^{n}+m_{\alpha}\right) D\left(\boldsymbol{v}^{n}\right), A \boldsymbol{v}^{n}\right)
\end{aligned}
$$

Taking into account that

$$
\begin{aligned}
-\left(\nu\left(\eta^{n}+m_{\alpha}\right) \nabla \bar{q}_{m}, A \boldsymbol{v}^{n}\right) & =\left(\bar{q}_{m}, \nu^{\prime}\left(\eta^{n}+m_{\alpha}\right) \nabla\left(\eta^{n}+m_{\alpha}\right) A \boldsymbol{v}^{n}\right)+\left(\bar{q}_{m}, \nu\left(\eta^{n}+m_{\alpha}\right) \nabla \cdot A \boldsymbol{v}^{n}\right) \\
& =\left(\bar{q}_{m}, \nu^{\prime}\left(\eta^{n}+m_{\alpha}\right) \nabla\left(\eta^{n}+m_{\alpha}\right) A \boldsymbol{v}^{n}\right)
\end{aligned}
$$

since $\nabla \cdot A \boldsymbol{v}^{n}=0$, the second term of (20) becomes

$$
\begin{aligned}
-2\left(\nabla \cdot\left(\nu\left(\eta^{n}+m_{\alpha}\right) D\left(\boldsymbol{v}^{n}\right)\right), A \boldsymbol{v}^{n}\right) & =\left(\nu\left(\eta^{n}+m_{\alpha}\right) A \boldsymbol{v}^{n}, A \boldsymbol{v}^{n}\right) \\
& -\left(\bar{q}_{m}, \nu^{\prime}\left(\eta^{n}+m_{\alpha}\right) \nabla\left(\eta^{n}+m_{\alpha}\right) A \boldsymbol{v}^{n}\right) \\
& -2\left(\nu^{\prime}\left(\eta^{n}+m_{\alpha}\right) \nabla\left(\eta^{n}+m_{\alpha}\right) D\left(\boldsymbol{v}^{n}\right), A \boldsymbol{v}^{n}\right) .
\end{aligned}
$$

Then, proceeding in analogous way for third and fourth term, (20) can also be written as follows (using $\nu(\cdot) \geq \nu_{\min }>0$ )

$$
\begin{aligned}
\frac{1}{2} \frac{d}{d t} \mid & \left.D\left(\boldsymbol{v}^{n}\right)\right|^{2}+\nu_{\min }\left\|\boldsymbol{v}^{n}\right\|_{2}^{2} \leq\left(\bar{q}_{m}, \nu^{\prime}\left(\eta^{n}+m_{\alpha}\right) \nabla\left(\eta^{n}+m_{\alpha}\right) A \boldsymbol{v}^{n}\right) \\
& +2\left(\nu^{\prime}\left(\eta^{n}+m_{\alpha}\right) \nabla\left(\eta^{n}+m_{\alpha}\right) D\left(\boldsymbol{v}^{n}\right), A \boldsymbol{v}^{n}\right)-\left(\nu\left(\eta^{n}+m_{\alpha}\right) A \boldsymbol{u}_{\alpha}, A \boldsymbol{v}^{n}\right) \\
& \left(q_{\alpha}, \nu^{\prime}\left(\eta^{n}+m_{\alpha}\right) \nabla\left(\eta^{n}+m_{\alpha}\right) A \boldsymbol{v}^{n}\right)+2\left(\nu^{\prime}\left(\eta^{n}+m_{\alpha}\right) \nabla\left(\eta^{n}+m_{\alpha}\right) D\left(\boldsymbol{u}_{\alpha}\right), A \boldsymbol{v}^{n}\right) \\
& +\left(\nu\left(m_{\alpha}\right) A \boldsymbol{u}_{\alpha}, A \boldsymbol{v}^{n}\right)-\left(q_{\alpha}, \nu^{\prime}\left(m_{\alpha}\right) \nabla m_{\alpha} A \boldsymbol{v}^{n}\right)-2\left(\nu^{\prime}\left(m_{\alpha}\right) \nabla m_{\alpha} D\left(\boldsymbol{u}_{\alpha}\right), A \boldsymbol{v}^{n}\right) \\
& -\left(\left(\boldsymbol{v}^{n} \cdot \nabla\right) \boldsymbol{v}^{n}, A \boldsymbol{v}^{n}\right)-\left(\left(\boldsymbol{u}_{\alpha} \cdot \nabla\right) \boldsymbol{v}^{n}, A \boldsymbol{v}^{n}\right)-\left(\left(\boldsymbol{v}^{n} \cdot \nabla\right) \boldsymbol{u}_{\alpha}, A \boldsymbol{v}^{n}\right)-\left(\eta^{n} \chi, A \boldsymbol{v}^{n}\right) \\
& :=\sum_{i=1}^{12} I_{i} .
\end{aligned}
$$


For to estimate the first term we use Lemma 8 (and $\left|\nu^{\prime}(\cdot)\right| \leq \nu_{\max }^{\prime}$ )

$$
\begin{aligned}
I_{1} & \leq \nu_{\text {max }}^{\prime}\left|q_{m}\right|_{3}\left(\left|\nabla \eta^{n}\right|_{6}+\left|\nabla m_{\alpha}\right|_{6}\right)\left|A \boldsymbol{v}^{n}\right|_{2} \leq C\left|q_{m}\right|_{2}^{1 / 2}\left\|q_{m}\right\|_{1}^{1 / 2}\left(\left\|\eta^{n}\right\|_{2}+\left\|m_{\alpha}\right\|_{2}\right)\left\|\boldsymbol{v}^{n}\right\|_{2} \\
& \leq C\left(C_{\varepsilon}\left\|\boldsymbol{v}^{n}\right\|_{1}^{1 / 2}+\varepsilon\left\|\boldsymbol{v}^{n}\right\|_{2}^{1 / 2}\right)\left\|\boldsymbol{v}^{n}\right\|_{2}^{3 / 2}\left(\left\|\eta^{n}\right\|_{2}+\left\|m_{\alpha}\right\|_{2}\right) \\
& \leq C_{\varepsilon}\left\|\boldsymbol{v}^{n}\right\|_{1}^{1 / 2}\left\|\boldsymbol{v}^{n}\right\|_{2}^{3 / 2}\left\|\left(\left\|\eta^{n}\right\|_{2}+\left\|m_{\alpha}\right\|_{2}\right)\right\|_{2}+\varepsilon C\left\|\boldsymbol{v}^{n}\right\|_{2}^{2}\left(\left\|\eta^{n}\right\|_{2}+\left\|m_{\alpha}\right\|_{2}\right) \\
& \leq \frac{\nu_{\min }}{28}\left\|\boldsymbol{v}^{n}\right\|_{2}^{2}+\frac{C}{\nu_{\min }}\left\|\boldsymbol{v}^{n}\right\|_{1}\left\|\boldsymbol{v}^{n}\right\|_{2}\left(\left\|\eta^{n}\right\|_{2}^{2}+\left\|m_{\alpha}\right\|_{2}^{2}\right)+\varepsilon C\left\|\boldsymbol{v}^{n}\right\|_{2}^{2}\left(\left\|\eta^{n}\right\|_{2}+\left\|m_{\alpha}\right\|_{2}\right) .
\end{aligned}
$$

Analogously,

$$
\begin{aligned}
I_{4} & \leq C\left(C_{\varepsilon}\left\|\boldsymbol{u}_{\alpha}\right\|_{1}^{1 / 2}+\varepsilon\left\|\boldsymbol{u}_{\alpha}\right\|_{2}^{1 / 2}\right)\left\|\boldsymbol{u}_{\alpha}\right\|_{2}^{1 / 2}\left(\left\|\eta^{n}\right\|_{2}+\left\|m_{\alpha}\right\|_{2}\right)\left\|\boldsymbol{v}^{n}\right\|_{2} \\
& \leq \frac{\nu_{\min }}{28}\left\|\boldsymbol{v}^{n}\right\|_{2}^{2}+\frac{C}{\nu_{\min }}\left\|\boldsymbol{u}_{\alpha}\right\|_{2}^{2}\left(\left\|\eta^{n}\right\|_{2}^{2}+\left\|m_{\alpha}\right\|_{2}^{2}\right) .
\end{aligned}
$$

and

$$
I_{7} \leq \frac{\nu_{\min }}{28}\left\|\boldsymbol{v}^{n}\right\|_{2}^{2}+\frac{C}{\nu_{\min }}\left\|\boldsymbol{u}_{\alpha}\right\|_{2}^{2}\left\|m_{\alpha}\right\|_{2}^{2} .
$$

The second term on the right hand side of (22) is bounded by

$$
\begin{aligned}
I_{2} & \leq 2 \nu_{\text {max }}^{\prime}\left(\left|\nabla \eta^{n}\right|_{6}+\left|\nabla m_{\alpha}\right|_{6}\right)\left|D\left(\boldsymbol{v}^{n}\right)\right|_{3}\left|A \boldsymbol{v}^{n}\right|_{2} \leq C\left(\left\|\eta^{n}\right\|_{2}+\left\|m_{\alpha}\right\|_{2}\right)\left\|\boldsymbol{v}^{n}\right\|_{1}^{1 / 2}\left\|\boldsymbol{v}^{n}\right\|_{2}^{3 / 2} \\
& \leq \frac{\nu_{\text {min }}}{28}\left\|\boldsymbol{v}^{n}\right\|_{2}^{2}+\frac{C}{\nu_{\text {min }}}\left(\left\|\eta^{n}\right\|_{2}^{2}+\left\|m_{\alpha}\right\|_{2}^{2}\right)\left\|\boldsymbol{v}^{n}\right\|_{1}\left\|\boldsymbol{v}^{n}\right\|_{2} .
\end{aligned}
$$

Analogously

$$
I_{5} \leq C\left(\left\|\eta^{n}\right\|_{2}+\left\|m_{\alpha}\right\|_{2}\right)\left\|\boldsymbol{u}_{\alpha}\right\|_{1}^{1 / 2}\left\|\boldsymbol{u}_{\alpha}\right\|_{2}^{1 / 2}\left\|\boldsymbol{v}^{n}\right\|_{2} \leq \frac{\nu_{\min }}{28}\left\|\boldsymbol{v}^{n}\right\|_{2}^{2}+\frac{C}{\nu_{\min }}\left(\left\|\eta^{n}\right\|_{2}^{2}+\left\|m_{\alpha}\right\|_{2}^{2}\right)\left\|\boldsymbol{u}_{\alpha}\right\|_{2}^{2} .
$$

and

$$
I_{8} \leq \frac{\nu_{\min }}{28}\left\|\boldsymbol{v}^{n}\right\|_{2}^{2}+\frac{C}{\nu_{\min }}\left\|m_{\alpha}\right\|_{2}^{2}\left\|\boldsymbol{u}_{\alpha}\right\|_{2}^{2} .
$$

The third and sixth terms are bounded by

$$
I_{i} \leq \frac{\nu_{\min }}{28}\left\|\boldsymbol{v}^{n}\right\|_{2}^{2}+\frac{C}{\nu_{\min }}\left\|\boldsymbol{u}_{\alpha}\right\|_{2}^{2} \quad i=3,6 .
$$

The ninth, tenth, eleventh terms and the last one term on the right hand side of (22) are bounded respectively by

$$
\begin{gathered}
I_{9} \leq \frac{\nu_{\min }}{28}\left\|\boldsymbol{v}^{n}\right\|_{2}^{2}+\frac{C}{\nu_{\min }}\left\|\boldsymbol{v}^{n}\right\|_{1}^{2}\left\|\boldsymbol{v}^{n}\right\|_{2}^{2}, \quad I_{10} \leq \frac{\nu_{\min }}{28}\left\|\boldsymbol{v}^{n}\right\|_{2}^{2}+\frac{C}{\nu_{\min }}\left\|\boldsymbol{u}_{\alpha}\right\|_{1}^{2}\left\|\boldsymbol{v}^{n}\right\|_{2}^{2}, \\
I_{11} \leq \frac{\nu_{\min }}{28}\left\|\boldsymbol{v}^{n}\right\|_{2}^{2}+\frac{C}{\nu_{\min }}\left\|\boldsymbol{u}_{\alpha}\right\|_{1}^{2} \quad \text { and } \quad I_{12} \leq \frac{\nu_{\text {min }}}{28}\left\|\boldsymbol{v}^{n}\right\|_{2}^{2}+\frac{C}{\nu_{\min }}\left|\eta^{n}\right|_{2}^{2} .
\end{gathered}
$$

Consequently, from (22) we arrive at

$$
\begin{gathered}
\frac{d}{d t}\left\|\boldsymbol{v}^{n}\right\|_{1}^{2}+\frac{4}{7} \nu_{\min }\left\|\boldsymbol{v}^{n}\right\|_{2}^{2} \leq \frac{C}{\nu_{\min }}\left[\left\|\boldsymbol{v}^{n}\right\|_{2}^{2}\left(\left\|\boldsymbol{v}^{n}\right\|_{1}^{2}+\left\|\eta^{n}\right\|_{2}^{2}+\left\|\boldsymbol{u}_{\alpha}\right\|_{2}^{2}+\left\|m_{\alpha}\right\|_{2}^{2}\right)\right. \\
\left.+\left\|\eta^{n}\right\|_{2}^{2}\left(\left\|\boldsymbol{u}_{\alpha}\right\|_{2}^{2}+1\right)+\left\|u_{\alpha}\right\|_{2}^{2}\left(\left\|m_{\alpha}\right\|_{2}^{2}+1\right)\right]+\varepsilon\left\|\boldsymbol{v}^{n}\right\|_{2}^{2}\left(\left\|\eta^{n}\right\|_{2}+\left\|m_{\alpha}\right\|_{2}\right) .
\end{gathered}
$$


On the other hand, using $\partial_{t} \boldsymbol{v}^{n}$ as a test function in the $\boldsymbol{v}^{n}$-system (10), one obtains

$$
\begin{aligned}
& \left(\partial_{t} \boldsymbol{v}^{n}, \partial_{t} \boldsymbol{v}^{n}\right)+2\left(\nu\left(\eta^{n}+m_{\alpha}\right) D\left(\boldsymbol{v}^{n}\right), \partial_{t} D\left(\boldsymbol{v}^{n}\right)\right)-2\left(\nabla \cdot\left(\nu\left(\eta^{n}+m_{\alpha}\right) D\left(\boldsymbol{u}_{\alpha}\right)\right), \partial_{t} \boldsymbol{v}^{n}\right) \\
& \quad+2\left(\nabla \cdot\left(\nu\left(m_{\alpha}\right) D\left(\boldsymbol{u}_{\alpha}\right)\right), \partial_{t} \boldsymbol{v}^{n}\right)+\left(\left(\boldsymbol{v}^{n} \cdot \nabla\right) \boldsymbol{v}^{n}, \partial_{t} \boldsymbol{v}^{n}\right)+\left(\left(\boldsymbol{v}^{n} \cdot \nabla\right) \boldsymbol{u}_{\alpha}, \partial_{t} \boldsymbol{v}^{n}\right) \\
& \quad=\left(\left(\boldsymbol{u}_{\alpha} \cdot \nabla\right) \boldsymbol{v}^{n}, \partial_{t} \boldsymbol{v}^{n}\right)=-\left(\eta^{n} \chi, \partial_{t} \boldsymbol{v}^{n}\right) .
\end{aligned}
$$

By taking into account that the second term of the left hand side of (24) can be written as $2\left(\nu\left(\eta^{n}+m_{\alpha}\right) D\left(\boldsymbol{v}^{n}\right), \partial_{t} D\left(\boldsymbol{v}^{n}\right)\right)=\frac{d}{d t}\left(\nu\left(\eta^{n}+m_{\alpha}\right) D\left(\boldsymbol{v}^{n}\right), D\left(\boldsymbol{v}^{n}\right)\right)-\left(\nu^{\prime}\left(\eta^{n}+m_{\alpha}\right) \partial_{t} \eta^{n} D\left(\boldsymbol{v}^{n}\right), D\left(\boldsymbol{v}^{n}\right)\right)$, we deduce from $(24)$ that

$$
\begin{aligned}
& \frac{d}{d t} \int_{\Omega} \nu\left(\eta^{n}+m_{\alpha}\right)\left|D\left(\boldsymbol{v}^{n}\right)\right|^{2}+\left|\partial_{t} \boldsymbol{v}^{n}\right|_{2}^{2} \leq\left(\nu^{\prime}\left(\eta^{n}+m_{\alpha}\right) \partial_{t} \eta^{n} D\left(\boldsymbol{v}^{n}\right), D\left(\boldsymbol{v}^{n}\right)\right) \\
& \quad+2\left(\nu^{\prime}\left(\eta^{n}+m_{\alpha}\right) \nabla\left(\eta^{n}+m_{\alpha}\right) D\left(\boldsymbol{u}_{\alpha}\right), \partial_{t} \boldsymbol{v}^{n}\right)+2\left(\nu\left(\eta^{n}+m_{\alpha}\right) \Delta \boldsymbol{u}_{\alpha}, \partial_{t} \boldsymbol{v}^{n}\right) \\
& \quad+2\left(\nu^{\prime}\left(m_{\alpha}\right) \nabla m_{\alpha} D\left(\boldsymbol{u}_{\alpha}\right), \partial_{t} \boldsymbol{v}^{n}\right)+2\left(\nu\left(m_{\alpha}\right) \Delta \boldsymbol{u}_{\alpha}, \partial_{t} \boldsymbol{v}^{n}\right) \\
& \quad-\left(\left(\boldsymbol{v}^{n} \cdot \nabla\right) \boldsymbol{v}^{n}, \partial_{t} \boldsymbol{v}^{n}\right)-\left(\left(\boldsymbol{v}^{n} \cdot \nabla\right) \boldsymbol{u}_{\alpha}, \partial_{t} \boldsymbol{v}^{n}\right)-\left(\left(\boldsymbol{u}_{\alpha} \cdot \nabla\right) \boldsymbol{v}^{n}, \partial_{t} \boldsymbol{v}^{n}\right)-\left(\eta^{n} \chi, \partial_{t} \boldsymbol{v}^{n}\right) \\
& \quad:=\sum_{i=1}^{9} J_{i} .
\end{aligned}
$$

The first term on the right side of $(25)$ is bounded by

$$
\begin{aligned}
J_{1} & \leq \nu_{\text {max }}^{\prime}\left|\partial_{t} \eta^{n}\right|_{2}\left|D\left(\boldsymbol{v}^{n}\right)\right|_{6}\left|D\left(\boldsymbol{v}^{n}\right)\right|_{3} \leq C\left|\partial_{t} \eta^{n}\right|_{2}\left\|\boldsymbol{v}^{n}\right\|_{2}^{3 / 2}\left\|\boldsymbol{v}^{n}\right\|_{1}^{1 / 2} \\
& \leq \frac{\nu_{\min }}{28}\left\|\boldsymbol{v}^{n}\right\|_{2}^{2}+\frac{C}{\nu_{\min }}\left|\partial_{t} \eta^{n}\right|_{2}^{2}\left\|\boldsymbol{v}^{n}\right\|_{1}\left\|\boldsymbol{v}^{n}\right\|_{2} .
\end{aligned}
$$

The second one is bounded by

$$
\begin{aligned}
J_{2} & \leq \nu_{\text {max }}^{\prime}\left|\nabla\left(\eta^{n}+m_{\alpha}\right)\right|_{6}\left|D\left(\boldsymbol{u}_{\alpha}\right)\right|_{3}\left|\partial_{t} \boldsymbol{v}^{n}\right|_{2} \leq C\left(\left\|\eta^{n}\right\|_{2}+\left\|m_{\alpha}\right\|_{2}\right)\left\|\boldsymbol{u}_{\alpha}\right\|_{2}\left|\partial_{t} \boldsymbol{v}^{n}\right|_{2} \\
& \leq \frac{1}{14}\left|\partial_{t} \boldsymbol{v}^{n}\right|_{2}^{2}+C\left\|\boldsymbol{u}_{\alpha}\right\|_{2}^{2}\left(\left\|\eta^{n}\right\|_{2}^{2}+\left\|m_{\alpha}\right\|_{2}^{2}\right)
\end{aligned}
$$

and analogously

$$
J_{4} \leq \frac{1}{14}\left|\partial_{t} \boldsymbol{v}^{n}\right|_{2}^{2}+C\left\|\boldsymbol{u}_{\alpha}\right\|_{2}^{2}\left\|m_{\alpha}\right\|_{2}^{2}
$$

It easy to see that the remaining terms are bounded respectively, by

$$
J_{i} \leq \frac{1}{14}\left|\partial_{t} \boldsymbol{v}^{n}\right|_{2}^{2}+C\left\|\boldsymbol{u}_{\alpha}\right\|_{2}^{2} \quad i=3,5,
$$

$J_{6} \leq \frac{1}{14}\left|\partial_{t} \boldsymbol{v}^{n}\right|_{2}^{2}+\frac{\nu_{\min }}{28}\left\|\boldsymbol{v}^{n}\right\|_{2}^{2}+\frac{C}{\nu_{\min }}\left|\boldsymbol{v}^{n}\right|_{2}^{2}\left\|\boldsymbol{v}^{n}\right\|_{1}^{2}\left\|\boldsymbol{v}^{n}\right\|_{2}^{2}, \quad J_{i} \leq \frac{1}{14}\left|\partial_{t} \boldsymbol{v}^{n}\right|_{2}^{2}+C\left\|\boldsymbol{u}_{\alpha}\right\|_{2}^{2}\left\|\boldsymbol{v}^{n}\right\|_{1}^{2} \quad i=7,8$ and

$$
J_{9} \leq \frac{1}{14}\left|\partial_{t} \boldsymbol{v}^{n}\right|_{2}^{2}+C\left|\eta^{n}\right|_{2}^{2}
$$


Consequently,

$$
\begin{aligned}
& \frac{d}{d t} \int_{\Omega} \nu\left(\eta^{n}+m_{\alpha}\right)\left|D\left(\boldsymbol{v}^{n}\right)\right|^{2}+\frac{1}{2}\left|\partial_{t} \boldsymbol{v}^{n}\right|_{2}^{2} \leq \frac{\nu_{\min }}{14}\left\|\boldsymbol{v}^{n}\right\|_{2}^{2}+\frac{C}{\nu_{\min }}\left\|\boldsymbol{v}^{n}\right\|_{2}^{2}\left(\left|\partial_{t} \eta^{n}\right|_{2}^{2}+\left\|\boldsymbol{v}^{n}\right\|_{1}^{2}\right) \\
& +C\left(\left\|\boldsymbol{v}^{n}\right\|_{1}^{2}\left\|\boldsymbol{u}_{\alpha}\right\|_{1}^{2}+\left\|\eta^{n}\right\|_{2}^{2}\left(\left\|\boldsymbol{u}_{\alpha}\right\|_{2}^{2}+1\right)+\left\|\boldsymbol{u}_{\alpha}\right\|_{2}^{2}\left(\left\|m_{\alpha}\right\|_{2}^{2}+1\right)\right) .
\end{aligned}
$$

Finally, (23) and (26) prove the Lemma.

Lemma 15 There exists $C>0$ such that

$$
\begin{aligned}
& \frac{d}{d t}\left(\left\|\eta^{n}\right\|_{2}^{2}+\left|\partial_{t} \eta^{n}\right|_{2}^{2}\right)+\theta\left\|\eta^{n}\right\|_{3}^{2}+\frac{\theta}{2}\left\|\partial_{t} \eta^{n}\right\|_{1}^{2} \leq \frac{\nu_{\min }}{4}\left\|\boldsymbol{v}^{n}\right\|_{2}^{2}+\frac{1}{4}\left|\partial_{t} \boldsymbol{v}^{n}\right|_{2}^{2} \\
& \quad+\frac{C}{\nu_{\min }}\left\|\eta^{n}\right\|_{3}^{2}\left(\left\|\eta^{n}\right\|_{2}+\left\|m_{\alpha}\right\|_{2}^{2}+\left\|\boldsymbol{v}^{n}\right\|_{1}^{\frac{2+4 \delta}{3+2 \delta}}\left\|\eta^{n}\right\|_{2}^{\frac{1-2 \delta}{3+2 \delta}}\right) \\
& \quad+\frac{C}{\nu_{\min }}\left\|\boldsymbol{v}^{n}\right\|_{1}^{2}\left(\left\|\eta^{n}\right\|_{2}^{4}+\left\|m_{\alpha}\right\|_{2}^{4}\right)+C\left|\partial_{t} \eta^{n}\right|_{2}^{2}\left(\left\|\eta^{n}\right\|_{2}^{4}+\left\|m_{\alpha}\right\|_{2}^{4}+1\right) \\
& \quad+C\left\|\eta^{n}\right\|_{2}^{2}\left(\left\|\boldsymbol{u}_{\alpha}\right\|_{1}^{4}+\left\|\boldsymbol{u}_{\alpha}\right\|_{1}^{\frac{4}{3-2 \delta}}+\left\|\boldsymbol{u}_{\alpha}\right\|_{2}^{\frac{8}{5-6 \delta}}+1\right)+C\left|\partial_{t} \eta^{n}\right|_{2}\left\|\eta^{n}\right\|_{2} .
\end{aligned}
$$

Proof. Differentiating respect to the time the $\eta^{n}$-equation of (11) and multiplying by $\partial_{t} \eta^{n}$ as test function, using that

$$
\left(\boldsymbol{v}^{n} \cdot \nabla \partial_{t} \eta^{n}, \partial_{t} \eta^{n}\right)=0, \quad\left(\boldsymbol{u}_{\alpha} \cdot \nabla \partial_{t} \eta^{n}, \partial_{t} \eta^{n}\right)=0
$$

and

$$
\theta\left(\nabla \partial_{t} \boldsymbol{v}^{n} \eta^{n} \cdot \mathbf{n}, \partial_{t} \eta^{n}\right)-U\left(\partial_{t} \eta^{n}, \partial_{t} \eta^{n} \cdot \mathbf{n}_{3}\right)=0 \quad \text { on } \quad(0, T) \times \partial \Omega
$$

one obtains

$$
\frac{1}{2} \frac{d}{d t}\left|\partial_{t} \eta^{n}\right|_{2}^{2}+\theta\left|\nabla \partial_{t} \eta^{n}\right|^{2}=-\left(\partial_{t} \boldsymbol{v}^{n} \nabla \eta^{n}, \partial_{t} \eta^{n}\right)-\left(\partial_{t} \boldsymbol{v}^{n} \nabla m_{\alpha}, \partial_{t} \eta^{n}\right)+U\left(\partial_{t} \eta^{n}, \frac{\partial}{\partial x_{3}}\left(\partial_{t} \eta^{n}\right)\right) .
$$

By bounding the two first terms on the right hand side of (28) in analogous way:

$$
\begin{aligned}
&-\left(\partial_{t} \boldsymbol{v}^{n} \nabla \eta^{n}, \partial_{t} \eta^{n}\right) \leq\left|\partial_{t} \boldsymbol{v}^{n}\right|_{2}\left|\nabla \eta^{n}\right|_{6}\left|\partial_{t} \eta^{n}\right|_{3} \leq C\left|\partial_{t} \boldsymbol{v}^{n}\right|_{2}\left\|\eta^{n}\right\|_{2}\left|\partial_{t} \eta^{n}\right|_{2}^{1 / 2}\left\|\partial_{t} \eta^{n}\right\|_{1}^{1 / 2} \\
& \leq \frac{1}{16}\left|\partial_{t} \boldsymbol{v}^{n}\right|_{2}^{2}+\frac{\theta}{6}\left\|\partial_{t} \eta^{n}\right\|_{1}^{2}+C\left\|\eta^{n}\right\|_{2}^{4}\left|\partial_{t} \eta^{n}\right|_{2}^{2} \\
&\left.-\left(\partial_{t} \boldsymbol{v}^{n} \nabla m_{\alpha}, \partial_{t} \eta^{n}\right) \leq \frac{1}{16}\left|\partial_{t} \boldsymbol{v}^{n}\right|_{2}^{2}+\frac{\theta}{6}\left\|\partial_{t} \eta^{n}\right\|_{1}^{2}\right)+C\left\|m_{\alpha}\right\|_{2}^{4}\left|\partial_{t} \eta^{n}\right|_{2}^{2}
\end{aligned}
$$

and the third one as

$$
U\left(\partial_{t} \eta^{n}, \frac{\partial}{\partial x_{3}}\left(\partial_{t} \eta^{n}\right)\right) \leq \frac{\theta}{6}\left\|\partial_{t} \eta^{n}\right\|_{1}^{2}+C\left|\partial_{t} \eta^{n}\right|_{2}^{2}
$$

we obtain

$$
\frac{d}{d t}\left|\partial_{t} \eta^{n}\right|_{2}^{2}+\theta\left\|\partial_{t} \eta^{n}\right\|_{1}^{2} \leq \frac{1}{4}\left|\partial_{t} \boldsymbol{v}^{n}\right|_{2}^{2}+C\left(\left\|\eta^{n}\right\|_{2}^{4}+\left\|m_{\alpha}\right\|_{2}^{4}+1\right)\left|\partial_{t} \eta^{n}\right|_{2}^{2}
$$


Note that $A_{1}^{2} \eta^{n} \in W^{n}$ owing to the choice of spectral basis, moreover $\nabla A_{1} \eta^{n} \cdot \mathbf{n}=\frac{U}{\theta} A_{1} \eta^{n} n_{3}$ on $\partial \Omega$ Therefore, by using $A_{1}^{2} \eta^{n}$ as test function, integrating by parts in all terms and taking into account that

$$
-\left(\nabla \partial_{t} \eta^{n}, \nabla A_{1}^{2} \eta^{n}\right)=\frac{1}{2} \frac{d}{d t}\left|A_{1} \eta^{n}\right|_{2}^{2}-\frac{U}{\theta} \int_{\partial \Omega} \partial_{t} \eta^{n} \mathrm{n}_{3} A_{1} \eta^{n} d S
$$

one obtains:

$$
\begin{aligned}
& \frac{1}{2} \frac{d}{d t}\left|A_{1} \eta^{n}\right|_{2}^{2}+\theta\left|\nabla A_{1} \eta^{n}\right|_{2}^{2}=\left(\nabla \boldsymbol{v}^{n} \nabla \eta^{n}, \nabla A_{1} \eta^{n}\right)+\left(\boldsymbol{v}^{n} \nabla^{2} \eta^{n}, \nabla A_{1} \eta^{n}\right)+\left(\nabla \boldsymbol{v}^{n} \nabla m_{\alpha}, \nabla A_{1} \eta^{n}\right) \\
& +\left(\boldsymbol{v}^{n} \nabla^{2} m_{\alpha}, \nabla A_{1} \eta^{n}\right)+\left(\nabla \boldsymbol{u}_{\alpha} \nabla \eta^{n}, \nabla A_{1} \eta^{n}\right)+\left(\boldsymbol{u}_{\alpha} \nabla^{2} \eta^{n}, \nabla A_{1} \eta^{n}\right)+U\left(\frac{\partial \nabla \eta^{n}}{\partial x_{3}}, \nabla A_{1} \eta^{n}\right) \\
& \quad+\frac{U}{\theta} \int_{\partial \Omega} \partial_{t} \eta^{n} \mathrm{n}_{3} A_{1} \eta^{n} d S-\int_{\partial \Omega} \partial_{t} \eta^{n} \nabla A_{1} \eta^{n} \mathbf{n} d S+\theta \int_{\partial \Omega} \Delta \eta^{n} \nabla A_{1} \eta^{n} \mathbf{n} d S \\
& \quad-\int_{\partial \Omega} \boldsymbol{v}^{n} \nabla \eta^{n} \nabla A_{1} \eta^{n} \mathbf{n} d S-\int_{\partial \Omega} \boldsymbol{v}^{n} \nabla m_{\alpha} \nabla A_{1} \eta^{n} \mathbf{n} d S-\int_{\partial \Omega} \boldsymbol{u}_{\alpha} \nabla \eta^{n} \nabla A_{1} \eta^{n} \mathbf{n} d S \\
& \quad-U \int_{\partial \Omega} \frac{\partial \eta^{n}}{\partial x_{3}} \nabla A_{1} \eta^{n} d S=\sum_{i=1}^{14} L_{i} .
\end{aligned}
$$

We will replace the following estimations for the $L_{i}, i=1,7$ in the above inequality:

$$
\begin{aligned}
& L_{1} \leq\left|\nabla \boldsymbol{v}^{n}\right|_{6}\left|\nabla \eta^{n}\right|_{3}\left|\nabla A_{1} \eta^{n}\right|_{2} \leq C\left\|\boldsymbol{v}^{n}\right\|_{2}\left\|\eta^{n}\right\|_{1}^{1 / 2}\left\|\eta^{n}\right\|_{2}^{1 / 2}\left\|\eta^{n}\right\|_{3} \\
& \leq \frac{\nu_{\min }}{48}\left\|\boldsymbol{v}^{n}\right\|_{2}^{2}+\frac{C}{\nu_{\min }}\left\|\eta^{n}\right\|_{1}\left\|\eta^{n}\right\|_{2}\left\|\eta^{n}\right\|_{3}^{2} . \\
& L_{2} \leq\left|\boldsymbol{v}^{n}\right|_{\infty}\left|\nabla^{2} \eta^{n}\right|_{2}\left|\nabla A_{1} \eta^{n}\right|_{2} \leq C\left\|\boldsymbol{v}^{n}\right\|_{1}^{1 / 2}\left\|\boldsymbol{v}^{n}\right\|_{2}^{1 / 2}\left\|\eta^{n}\right\|_{2}\left\|\eta^{n}\right\|_{3} \\
& \leq \frac{\nu_{\min }}{48}\left\|\boldsymbol{v}^{n}\right\|_{2}^{2}+\frac{\theta}{20}\left\|\eta^{n}\right\|_{3}^{2}++\frac{C}{\nu_{\min }}\left\|\boldsymbol{v}^{n}\right\|_{1}^{2}\left\|\eta^{n}\right\|_{2}^{4} . \\
& \leq\left|\nabla \boldsymbol{v}^{n}\right|_{6}\left|\nabla m_{\alpha}\right|_{3}\left|\nabla A_{1} \eta^{n}\right|_{2} \leq C\left\|\boldsymbol{v}^{n}\right\|_{2}\left\|m_{\alpha}\right\|_{2}\left\|\eta^{n}\right\|_{3} \\
& \leq \frac{\nu_{\min }}{48}\left\|\boldsymbol{v}^{n}\right\|_{2}^{2}+\frac{C}{\nu_{\min }}\left\|m_{\alpha}\right\|_{2}^{2}\left\|\eta^{n}\right\|_{3}^{2} . \\
& \leq\left|\boldsymbol{v}^{n}\right|_{\infty}\left|\nabla^{2} m_{\alpha}\right|_{2}\left|\nabla A_{1} \eta^{n}\right|_{2} \leq C\left\|\boldsymbol{v}^{n}\right\|_{1}^{1 / 2}\left\|\boldsymbol{v}^{n}\right\|_{2}^{1 / 2}\left\|m_{\alpha}\right\|_{2}\left\|\eta^{n}\right\|_{3} \\
& \leq \frac{\nu_{\min }}{48}\left\|\boldsymbol{v}^{n}\right\|_{2}^{2}+\frac{\theta}{20}\left\|\eta^{n}\right\|_{3}^{2}++\frac{C}{\nu_{\min }}\left\|\boldsymbol{v}^{n}\right\|_{1}^{2}\left\|m_{\alpha}\right\|_{2}^{4} . \\
& L_{5} \leq\left|\nabla \boldsymbol{u}_{\alpha}\right|_{6}\left|\nabla^{2} \eta^{n}\right|_{3}\left|\nabla A_{1} \eta^{n}\right|_{2} \leq C\left\|\boldsymbol{u}_{\alpha}\right\|_{2}\left\|\eta^{n}\right\|_{2}^{1 / 2}\left\|\eta^{n}\right\|_{3}^{3 / 2} \leq \frac{\theta}{20}\left\|\eta^{n}\right\|_{3}^{2}+C\left\|\boldsymbol{u}_{\alpha}\right\|_{1}^{4}\left\|\eta^{n}\right\|_{2}^{2} \\
& L_{6} \leq\left|\boldsymbol{u}_{\alpha}\right|_{6}\left|\nabla^{2} \eta^{n}\right|_{3}\left|\nabla A_{1} \eta^{n}\right|_{2} \leq C\left\|\boldsymbol{u}_{\alpha}\right\|_{1}\left\|\eta^{n}\right\|_{2}^{1 / 2}\left\|\eta^{n}\right\|_{3}^{3 / 2} \leq \frac{\theta}{20}\left\|\eta^{n}\right\|_{3}^{2}+C\left\|\boldsymbol{u}_{\alpha}\right\|_{1}^{4}\left\|\eta^{n}\right\|_{2}^{2} .
\end{aligned}
$$




$$
L_{7} \leq C\left\|\eta^{n}\right\|_{2}\left\|\eta^{n}\right\|_{3} \leq \frac{\theta}{20}\left\|\eta^{n}\right\|_{3}^{2}+C\left\|\eta^{n}\right\|_{2}^{2} .
$$

To bound boundary terms we use Lemma 5 and in some cases, the equality $\nabla A_{1} \eta^{n} \cdot \mathbf{n}=$ $\frac{U}{\theta} A_{1} \eta^{n} \mathrm{n}_{3}$, provided by the boundary conditions.

$$
\begin{aligned}
L_{i} \leq & \frac{U}{\theta}\left|\partial_{t} \eta^{n} \mathrm{n}_{3}\right|_{L^{2}(\partial \Omega)}\left|A_{1} \eta^{n}\right|_{L^{2}(\partial \Omega)} \leq C\left|\partial_{t} \eta^{n}\right|_{2}^{1 / 2-\delta}\left\|\partial_{t} \eta^{n}\right\|_{1}^{1 / 2+\delta}\left\|\eta^{n}\right\|_{2}^{1 / 2-\delta}\left\|\eta^{n}\right\|_{3}^{1 / 2+\delta} \\
\leq & \frac{\theta}{20}\left\|\eta^{n}\right\|_{3}^{2}+\frac{\theta}{8}\left\|\partial_{t} \eta^{n}\right\|_{1}^{2}+C\left\|\eta^{n}\right\|_{2}\left|\partial_{t} \eta^{n}\right|_{2} \quad \text { for } i=8,9 . \\
L_{10} \leq & \frac{U}{\theta}\left|\int_{\partial \Omega} \Delta \eta^{n} A_{1} \eta^{n} \mathrm{n}_{3} d S\right| \leq C\left|A_{1} \eta^{n}\right|_{L^{2}(\partial \Omega)}^{2} \leq C\left\|\eta^{n}\right\|_{2}^{1 / 2-\delta}\left\|\eta^{n}\right\|_{3}^{1 / 2+\delta} \leq \frac{\theta}{20}\left\|\eta^{n}\right\|_{3}^{2}+C\left\|\eta^{n}\right\|_{2}^{2} . \\
L_{11} \leq & \frac{U}{\theta}\left|\boldsymbol{v}^{n} \nabla \eta^{n}\right|_{L^{2}(\partial \Omega)}\left|A_{1} \eta^{n}\right|_{L^{2}(\partial \Omega)} \leq C\left|\boldsymbol{v}^{n} \nabla \eta^{n}\right|_{2}^{1 / 2-\delta}\left\|\boldsymbol{v}^{n} \nabla \eta^{n}\right\|_{1}^{1 / 2+\delta}\left\|\eta^{n}\right\|_{2}^{1 / 2-\delta}\left\|\eta^{n}\right\|_{3}^{1 / 2+\delta} \\
\leq & C\left(\left\|\boldsymbol{v}^{n}\right\|_{1}\left\|\eta^{n}\right\|_{1}^{1 / 2}\left\|\eta^{n}\right\|_{2}^{1 / 2}\right)^{1 / 2-\delta}\left(\left\|\boldsymbol{v}^{n}\right\|_{2}\left\|\eta^{n}\right\|_{1}^{1 / 2}\left\|\eta^{n}\right\|_{2}^{1 / 2}+\left\|\boldsymbol{v}^{n}\right\|_{1}\left\|\eta^{n}\right\|_{2}^{1 / 2}\left\|\eta^{n}\right\|_{3}^{1 / 2}\right)^{1 / 2+\delta} \\
& \cdot\left\|\eta^{n}\right\|_{2}^{1 / 2-\delta}\left\|\eta^{n}\right\|_{3}^{1 / 2+\delta} \leq C\left(\left\|\boldsymbol{v}^{n}\right\|_{1}^{1 / 2-\delta}\left\|\boldsymbol{v}^{n}\right\|_{2}^{1 / 2+\delta}\left\|\eta^{n}\right\|_{1}^{1 / 2}\left\|\eta^{n}\right\|_{2}^{1-\delta}\left\|\eta^{n}\right\|_{3}^{1 / 2+\delta}\right. \\
& \left.+\left\|\boldsymbol{v}^{n}\right\|_{1}\left\|\eta^{n}\right\|_{1}^{\frac{1}{4}-\frac{\delta}{2}}\left\|\eta^{n}\right\|_{2}^{1-\delta}\left\|\eta^{n}\right\|_{3}^{\frac{3}{4}+\frac{3 \delta}{2}}\right) \leq \frac{\nu_{\min }}{96}\left\|\boldsymbol{v}^{n}\right\|_{2}^{2}+\frac{C}{\nu_{\min }}\left\|\eta^{n}\right\|_{1}\left\|\eta^{n}\right\|_{2}^{2-2 \delta}\left\|\eta^{n}\right\|_{3}^{1+2 \delta} \\
& +\frac{\nu_{\min }}{96}\left\|\boldsymbol{v}^{n}\right\|_{1}^{2}+\frac{C}{\nu_{\min }}\left\|\boldsymbol{v}^{n}\right\|_{1}^{\frac{2+4 \delta}{3+2 \delta}}\left\|\eta^{n}\right\|_{2}^{\frac{4-4 \delta}{3+2 \delta}}\left\|\eta^{n}\right\|_{3}^{\frac{3+6 \delta}{3+2 \delta}} \\
\leq & \frac{\nu_{\min }}{48}\left\|\boldsymbol{v}^{n}\right\|_{2}^{2}+\frac{C}{\nu_{\min }}\left\|\eta^{n}\right\|_{2}\left\|\eta^{n}\right\|_{3}^{2}+\left\|\boldsymbol{v}^{n}\right\|_{1}^{\frac{2+4 \delta}{3+2 \delta}}\left\|\eta^{n}\right\|_{2}^{\frac{1-2 \delta}{3+2 \delta}}\left\|\eta^{n}\right\|_{3}^{2} .
\end{aligned}
$$

$$
\begin{aligned}
L_{12} \leq & C\left(\left\|\boldsymbol{v}^{n}\right\|_{1}\left\|m_{\alpha}\right\|_{1}^{1 / 2}\left\|m_{\alpha}\right\|_{2}^{1 / 2}\right)^{1 / 2-\delta}\left(\left\|\boldsymbol{v}^{n}\right\|_{2}\left\|m_{\alpha}\right\|_{1}^{1 / 2}\left\|m_{\alpha}\right\|_{2}^{1 / 2}+\left\|\boldsymbol{v}^{n}\right\|_{1}^{1 / 2}\left\|\boldsymbol{v}^{n}\right\|_{2}^{1 / 2}\left\|m_{\alpha}\right\|_{2}\right)^{1 / 2+\delta} \\
& \cdot\left\|\eta^{n}\right\|_{2}^{1 / 2-\delta}\left\|\eta^{n}\right\|_{3}^{1 / 2+\delta} \leq\left\|\boldsymbol{v}^{n}\right\|_{1}^{\frac{1-2 \delta}{2}}\left\|m_{\alpha}\right\|_{2}\left\|\boldsymbol{v}^{n}\right\|_{2}^{\frac{1+2 \delta}{2}}\left\|\eta^{n}\right\|_{2}^{\frac{1-2 \delta}{2}}\left\|\eta^{n}\right\|_{3}^{\frac{1+2 \delta}{2}} \\
& +\left\|\boldsymbol{v}^{n}\right\|_{1}^{\frac{3-2 \delta}{4}}\left\|\boldsymbol{v}^{n}\right\|_{2}^{\frac{1+2 \delta}{4}}\left\|m_{\alpha}\right\|_{2}\left\|\boldsymbol{v}^{n}\right\|_{2}^{\frac{1+2 \delta}{2}}\left\|\eta^{n}\right\|_{2}^{\frac{1-2 \delta}{2}}\left\|\eta^{n}\right\|_{3}^{\frac{1+2 \delta}{2}} \\
\leq & \frac{\nu_{\min }}{48}\left\|\boldsymbol{v}^{n}\right\|_{2}^{2}+\frac{C}{\nu_{\min }}\left\|m_{\alpha}\right\|_{2}^{2}\left\|\eta^{n}\right\|_{3}^{2} .
\end{aligned}
$$

$$
\begin{gathered}
L_{13} \leq \quad C\left(\left\|\boldsymbol{u}_{\alpha}\right\|_{1}\left\|\eta^{n}\right\|_{1}^{1 / 2}\left\|\eta^{n}\right\|_{2}^{1 / 2}\right)^{1 / 2-\delta}\left(\left\|\boldsymbol{u}_{\alpha}\right\|_{2}\left\|\eta^{n}\right\|_{1}^{1 / 2}\left\|\eta^{n}\right\|_{2}^{1 / 2}+\left\|\boldsymbol{u}_{\alpha}\right\|_{1}\left\|\eta^{n}\right\|_{2}^{1 / 2}\left\|\eta^{n}\right\|_{3}^{1 / 2}\right)^{1 / 2+\delta} \\
\cdot\left\|\eta^{n}\right\|_{2}^{1 / 2-\delta}\left\|\eta^{n}\right\|_{3}^{1 / 2+\delta} \leq \frac{\theta}{20}\left\|\eta^{n}\right\|_{3}^{2} \\
+C\left(\left\|\boldsymbol{u}_{\alpha}\right\|_{1}^{\frac{2-4 \delta}{3-2 \delta}}\left\|\boldsymbol{u}_{\alpha}\right\|_{2}^{\frac{2+4 \delta}{3-2 \delta}}\left\|\eta^{n}\right\|_{1}^{\frac{2}{3-2 \delta}}\left\|\eta^{n}\right\|_{2}^{\frac{4-4 \delta}{3-2 \delta}}+\left\|\boldsymbol{u}_{\alpha}\right\|_{1}^{\frac{8}{5-6 \delta}}\left\|\eta^{n}\right\|_{1}^{\frac{2-4 \delta}{5-6 \delta}}\left\|\eta^{n}\right\|_{2}^{\frac{8-8 \delta}{5-6 \delta}}\right) \\
\leq \frac{\theta}{20}\left\|\eta^{n}\right\|_{3}^{2}+C\left(\left\|\boldsymbol{u}_{\alpha}\right\|_{2}^{\frac{8}{5-6 \delta}}+\left\|\boldsymbol{u}_{\alpha}\right\|_{1}^{\frac{4}{3-2 \delta}}\right)\left\|\eta^{n}\right\|_{2}^{2} . \\
\leq C\left|\frac{\partial \eta^{n}}{\partial x_{3}}\right|_{2}^{1 / 2-\delta}\left\|\frac{\partial \eta^{n}}{\partial x_{3}}\right\|_{1}^{1 / 2+\delta}\left|A_{1} \eta^{n}\right|_{2}^{1 / 2-\delta}\left\|A_{1} \eta^{n}\right\|_{1}^{1 / 2+\delta} \\
\leq C\left\|\eta^{n}\right\|_{1}^{1 / 2-\delta}\left\|\eta^{n}\right\|_{2}\left\|\eta^{n}\right\|_{3}^{1 / 2+\delta} \leq \frac{\theta}{20}\left\|\eta^{n}\right\|_{3}^{2}+C\left\|^{n}\right\|_{2}^{2} .
\end{gathered}
$$


Then we get,

$$
\begin{aligned}
& \frac{d}{d t}\left\|\eta^{n}\right\|_{2}^{2}+\theta\left\|\eta^{n}\right\|_{3}^{2} \leq \frac{\nu_{\min }}{4}\left\|\boldsymbol{v}^{n}\right\|_{2}^{2}+\frac{\theta}{2}\left\|\partial_{t} \eta^{n}\right\|_{1}^{2}+\frac{C}{\nu_{\min }}\left\|\boldsymbol{v}^{n}\right\|_{1}^{2}\left(\left\|\eta^{n}\right\|_{2}^{4}+\left\|m_{\alpha}\right\|_{2}^{4}\right) \\
& \quad+\frac{C}{\nu_{\min }}\left\|\eta^{n}\right\|_{3}^{2}\left(\left\|\eta^{n}\right\|_{2}+\left\|m_{\alpha}\right\|_{2}^{2}+\left\|\boldsymbol{v}^{n}\right\|_{1}^{\frac{2+4 \delta}{3+2 \delta}}\left\|\eta^{n}\right\|_{2}^{\frac{1-2 \delta}{3+2 \delta}}\right) \\
& \quad+C\left\|\eta^{n}\right\|_{2}^{2}\left(\left\|\boldsymbol{u}_{\alpha}\right\|_{1}^{4}+\left\|\boldsymbol{u}_{\alpha}\right\|_{1}^{\frac{4}{3-2 \delta}}+\left\|\boldsymbol{u}_{\alpha}\right\|_{2}^{\frac{8}{5-6 \delta}}+1\right)+C\left|\partial_{t} \eta^{n}\right|_{2}\left\|\eta^{n}\right\|_{2} .
\end{aligned}
$$

Finally, (29) added to (30) proves the Lemma.

\section{$5 \quad$ Proof of Theorem 13}

If we suppose $\nu_{\min } \geq 1$ adding (19) and (27), one has

$$
\begin{aligned}
& \frac{d}{d t}\left(\int_{\Omega}\left(\nu\left(\eta^{n}+m_{\alpha}\right)+1\right)\left|D\left(\boldsymbol{v}^{n}\right)\right|^{2}+\left\|\eta^{n}\right\|_{2}^{2}+\left|\partial_{t} \eta^{n}\right|_{2}^{2}\right)+\frac{\nu_{\min }}{4}\left\|\boldsymbol{v}^{n}\right\|_{2}^{2}+\frac{1}{4}\left|\partial_{t} \boldsymbol{v}^{n}\right|_{2}^{2} \\
& \quad+\theta\left\|\eta^{n}\right\|_{3}^{2}+\frac{\theta}{2}\left\|\partial_{t} \eta^{n}\right\|_{1}^{2} \leq \varepsilon\left\|\boldsymbol{v}^{n}\right\|_{2}^{2}\left(\left\|\eta^{n}\right\|_{2}+\left\|m_{\alpha}\right\|_{2}\right) \\
& \quad+\frac{C}{\nu_{\min }}\left\|\boldsymbol{v}^{n}\right\|_{2}^{2}\left(\left\|\boldsymbol{v}^{n}\right\|_{1}^{2}+\left\|\eta^{n}\right\|_{2}^{2}+\left|\partial_{t} \eta^{n}\right|_{2}^{2}+\left\|\boldsymbol{u}_{\alpha}\right\|_{2}^{2}+\left\|m_{\alpha}\right\|_{2}^{2}\right) \\
& \quad+\frac{C}{\nu_{\min }}\left\|\eta^{n}\right\|_{3}^{2}\left(\left\|\eta^{n}\right\|_{2}+\left\|m_{\alpha}\right\|_{2}^{2}+\left\|\boldsymbol{v}^{n}\right\|_{1}^{\frac{2+4 \delta}{3+2 \delta}}\left\|\eta^{n}\right\|_{2}^{\frac{1-2 \delta}{3+2 \delta}}\right) \\
& \quad+C\left\|\eta^{n}\right\|_{2}^{2}\left(\left\|\boldsymbol{u}_{\alpha}\right\|_{1}^{4}+\left\|\boldsymbol{u}_{\alpha}\right\|_{1}^{2}+\left\|\boldsymbol{u}_{\alpha}\right\|_{1}^{\frac{4}{3-2 \delta}}+\left\|\boldsymbol{u}_{\alpha}\right\|_{2}^{\frac{8}{5-6 \delta}}+1\right) \\
& \quad+C\left\|\boldsymbol{v}^{n}\right\|_{1}^{2}\left(\left\|\eta^{n}\right\|_{2}^{4}+\left\|\boldsymbol{u}_{\alpha}\right\|_{1}^{2}+\left\|m_{\alpha}\right\|_{2}^{4}\right)+C\left|\partial_{t} \eta^{n}\right|_{2}\left\|\eta^{n}\right\|_{2} \\
& \quad+C\left|\partial_{t} \eta^{n}\right|_{2}^{2}\left(\left\|\eta^{n}\right\|_{2}^{4}+\left\|m_{\alpha}\right\|_{2}^{4}+1\right)+C\left\|\boldsymbol{u}_{\alpha}\right\|_{2}^{2}\left(\left\|m_{\alpha}\right\|_{2}^{2}+1\right) .
\end{aligned}
$$

By denoting

$$
\begin{gathered}
\Phi_{1}(t)=\int_{\Omega}\left(\nu\left(\eta^{n}+m_{\alpha}\right)+1\right)\left|D\left(\boldsymbol{v}^{n}\right)\right|^{2}, \quad \Phi_{2}=\left\|\eta^{n}\right\|_{2}^{2}+\left|\partial_{t} \eta^{n}\right|_{2}^{2}, \quad \Phi=\Phi_{1}+\Phi_{2}, \\
\Psi_{1}(t)=\left\|\boldsymbol{v}^{n}\right\|_{2}^{2} \quad \text { and } \quad \Psi_{2}=\left|\partial_{t} \boldsymbol{v}^{n}\right|_{2}^{2}+\left\|\eta^{n}\right\|_{3}^{2}+\left\|\partial_{t} \eta^{n}\right\|_{1}^{2}
\end{gathered}
$$

and taking into account that $\left\|\boldsymbol{u}_{\alpha}\right\|_{2},\left\|m_{\alpha}\right\|_{2}$ are bounded, one has

$$
\left\{\begin{array}{l}
\Phi^{\prime}+\frac{\nu_{\min }}{4} \Psi_{1}+\bar{C} \Psi_{2} \leq \varepsilon \Psi_{1}\left(\Phi_{2}^{1 / 2}+\left\|m_{\alpha}\right\|_{2}\right) \\
\quad+\frac{C_{1}}{\nu_{\min }} \Psi_{1}(\Phi+1)+\frac{C_{2}}{\nu_{\min }} \Psi_{2}\left(\Phi^{1 / 2}+\left\|m_{\alpha}\right\|_{2}^{2}\right)+D \Phi \Phi_{2}^{2}+E \\
\Phi(0)=\Phi_{0} .
\end{array}\right.
$$

where $C_{1}, C_{2}, D, E>0$ are constants independents of $\nu_{\min }$.

First step: We will prove that if $\Phi(0) \leq M / 2$, then $\Phi(t) \leq M \forall t \in[0, T]$, where $M$ is a positive constant that we will specify below. 
Let us define $R_{1}, R_{2}$ and $R_{3}$ as follows: $\|\boldsymbol{v}(0)\|_{1}=R_{1}, \Phi_{2}(0)=R_{2}$ and $\nu_{\max }-\nu_{\min }=R_{3}$. Assume that $\varepsilon, R_{1}$ and $R_{2}$ are small enough and $\nu_{\min }$ is big enough such that,

$$
\frac{\nu_{\min }}{4}-\varepsilon\left(\left(\left(\nu_{\min }+R_{3}+1\right) R_{1}+R_{2}\right)^{1 / 2}+\left\|m_{\alpha}\right\|_{2}\right)-\frac{C_{1}}{\nu_{\min }}\left(\left(\nu_{\min }+R_{3}+1\right) R_{1}+R_{2}+1\right) \geq a_{1}
$$

and

$$
\bar{C}-\frac{C_{2}}{\nu_{\min }}\left(\left(\left(\nu_{\min }+R_{3}+1\right) R_{1}+R_{2}\right)^{1 / 2}+\left\|m_{\alpha}\right\|_{2}^{2}\right) \geq a_{2},
$$

for some constants $a_{1}, a_{2}>0$. We define $a=\min \left\{a_{1}, a_{2}\right\}$ and $P=\min \left\{P_{1}, P_{2}\right\}$ where $1 / P_{1}$ and $1 / P_{2}$ are the Poincaré constants that verify $\Phi_{1} \leq \frac{1}{P_{1}} \Psi_{1}$ and $\Phi_{2} \leq \frac{1}{P_{2}} \Psi_{2}$ respectively.

Since in [3] one can see that

$$
\Phi(t) \leq \widetilde{C}\left(1+\frac{1}{\nu_{\min }}\right),
$$

we have that $\Phi(t) \leq 2 \widetilde{C}$ for all $t \in[0, T]$ where $\widetilde{C}$ is independent of $\nu_{\min }$. Now, we choose $\nu_{\min }$ so that (33) and (34) holds and

$$
C_{w}=\frac{8 D \widetilde{C}^{3}+E}{a P}<\left(\nu_{\min }+1\right) R_{1}+R_{2} .
$$

We put $M=M\left(\nu_{\max }, R_{1}, R_{2}\right)$ defined as $M / 2=\left(\nu_{\max }+1\right) R_{1}+R_{2}$. Let $t^{*}>0$ the first value such that $\Phi\left(t^{*}\right)=M$, hence

$$
\Phi\left(t^{*}\right)=M \quad \text { and } \quad \Phi(t)<M \quad \forall t \in\left[0, t^{*}\right) .
$$

The following differential inequality holds:

$$
\left\{\begin{array}{l}
\Phi^{\prime}+a P \Phi \leq D \Phi \Phi_{2}^{2}+E . \\
\Phi(0)=\Phi_{0}
\end{array}\right.
$$

for each $t \in\left[0, t^{*}\right]$.

Multiplying (36) by $e^{a P t}$ and integrating in $\left[0, t^{*}\right]$ we deduce

$$
\Phi\left(t^{*}\right) \leq \Phi(0) e^{-a P t^{*}}+e^{-a P t^{*}} \int_{0}^{t^{*}}\left(D \Phi \Phi_{2}^{2}+E\right) e^{a P s} d s .
$$

Therefore, by using $\Phi_{2} \in L^{\infty}, \Phi_{1} \in L^{1}$ and taking into account (35), we obtain

$$
\begin{aligned}
\Phi\left(t^{*}\right) & \leq \Phi(0) e^{-a P t^{*}}+\left(D(2 \widetilde{C})^{3}+E\right) \frac{1}{a P}\left(1-e^{-a P T}\right) \\
& \leq\left(\nu_{\max }+1\right)\|\boldsymbol{v}(0)\|_{1}^{2}+\Phi_{2}(0)+C_{w} \leq\left(\nu_{\max }+1\right) R_{1}+R_{2}+C_{w}
\end{aligned}
$$

but $C_{w}<M / 2$, then we arrives at a contradiction.

Second step: Under conditions of first step then $\Phi(T) \leq \Phi(0)$.

Now, since $\Phi(t)<M \forall t \in[0, T]$, we can repeat the above argument obtaining (36) in $[0, T]$ and we arrive at

$$
\Phi(T) \leq \Phi(0) e^{-a P T}+C_{w}
$$


Choosing $\nu_{\text {min }}$ big enough such that $C_{w} \leq\left(\left(\nu_{\min }+1\right) R_{1}+R_{2}\right)\left(1-e^{-a P T}\right)$ we obtain $\Phi(T) \leq M / 2$. Hence, $\Phi(T) \leq \Phi(0)$

Third step: Existence of approximate periodic solution

Given $\left(\boldsymbol{v}^{n 0}, \eta^{n 0}\right) \in V^{n} \times W^{n}$, we define the map

$$
\begin{aligned}
L^{n}:[0, T] & \mapsto \mathbb{R}^{n} \times \mathbb{R}^{n} \\
t & \mapsto\left(\xi_{1 n}(t), \ldots, \xi_{n n}(t), \zeta_{1 n}(t), \ldots, \zeta_{n n}(t)\right)
\end{aligned}
$$

where $\left(\xi_{1 n}(t), \ldots, \xi_{n n}(t)\right)$ and $\left(\zeta_{1 n}(t), \ldots, \zeta_{n n}(t)\right)$ are coefficients of $\boldsymbol{v}^{n}(t)$ and $\eta^{n}(t)$ respect to $V^{n}$ and $W^{n}$ respectively, being $\left(\boldsymbol{v}^{n}(t), \eta^{n}(t)\right)$ the (unique) approximate solution of (10)-(11) corresponding to the initial data $\left(\boldsymbol{v}_{n 0}, \theta_{n 0}\right)$.

Now, varying the initial data $\left(\boldsymbol{v}^{n 0}, \eta^{n 0}\right)$, we are going to define a new map

$$
\mathcal{R}^{n}: \bar{B} \subset \mathbb{R}^{n} \times \mathbb{R}^{n} \mapsto \mathbb{R}^{n} \times \mathbb{R}^{n}
$$

as follows: given $L_{0}^{n} \in \mathbb{R}^{n} \times \mathbb{R}^{n}$, we define $\mathcal{R}^{n}\left(L_{0}^{n}\right)=L^{n}(T)$, where $L^{n}(t)$ is related to the solution of problem $(10)-(11)$ with initial data $L_{0}^{n}\left(=L^{n}(0)\right)$ and

$$
\bar{B}=\left\{\left(\xi_{1 n}, \ldots, \xi_{n n}, \zeta_{1 n}, \ldots, \zeta_{n n}\right)=L_{0}^{n}: \Phi(0) \leq M / 2\right\} .
$$

By uniqueness of approximate solution of problem (10)-(11), this map is well-defined. Moreover, using regularity of the corresponding ordinary differential system (equivalent to (10)-(11)), this map is continuous. By the second step, $\mathcal{R}^{n}$ maps $\bar{B}$ into $\bar{B}$ and $\bar{B}$ is a closed, convex and compact set. Consequently, Brouwer Theorem implies the existence of fixed point of $\mathcal{R}^{n}$, which gives us existence of periodic Galerkin solution.

Four step: Pass to the limit in periodic approximate solutions

If the $\nu_{\min }$ is big, owing to the first step we have

$$
\Phi(t)=\int_{\Omega}\left(\nu\left(\eta^{n}+\left\|m_{\alpha}\right\|\right)+1\right)\left|\nabla \boldsymbol{v}^{n}\right|^{2}+\left\|\eta^{n}\right\|_{2}^{2}+\left|\partial_{t} \eta^{n}\right|_{2}^{2} \leq M .
$$

Therefore, the following bounds hold uniformly:

$$
\begin{gathered}
\left(\boldsymbol{v}^{n}, \eta^{n}\right) \text { in } L^{\infty}\left(\boldsymbol{H}^{1} \times H_{N}^{2}\right) \cap L^{2}\left(\boldsymbol{H}^{2} \times H_{N}^{3}\right), \\
\left(\partial_{t} \boldsymbol{v}^{n}\right) \text { in } L^{2}\left(\boldsymbol{L}^{2}\right), \\
\left(\partial_{t} \eta^{n}\right) \text { in } L^{\infty}\left(L^{2}\right) \cap L^{2}\left(H^{1}\right) .
\end{gathered}
$$

Using compactness results for time spaces with values in Banach spaces with the compact embedding of $H^{2}$ into $H^{1}$, one has

$$
\left(\boldsymbol{v}^{n}, \eta^{n}\right) \text { is relatively compact in } L^{2}\left(\boldsymbol{H}^{1} \times H^{2}\right) \text {. }
$$


In fact, this compactness is sufficient in the pass to the limit in the approximated problem (10), (11) in order to control the nonlinear terms.

Now, we go to pass to the limit in periodic conditions. From estimations of $\eta^{n}$ in $L^{\infty}\left(H^{2}\right)$ and $\left(\partial_{t} \eta^{n}\right)$ in $L^{2}\left(H^{1}\right)$ and using the compact embedding of $H^{2}$ into $H^{1}$, one has that $\eta^{n}$ is relatively compact in $C\left([0, T] ; H^{1}\right)$, hence $\eta^{n}(T) \rightarrow \eta(T)$ and $\eta^{n}(0) \rightarrow \eta(0)$ strongly in $H^{1}(\Omega)$. Since $\eta^{n}(T)=\eta^{n}(0)$, then $\eta(T)=\eta(0)$ in $H^{1}(\Omega)$. Finally, since $\eta^{n}(T)$ and $\eta^{n}(0)$ are bounded in $H^{2}(\Omega)$, we have that $\eta(T)=\eta(0)$ in $H^{2}(\Omega)$.

The argument for $\boldsymbol{u}$ is similar, hence one deduces $\boldsymbol{u}(T)=\boldsymbol{u}(0)$ in $\boldsymbol{H}^{1}(\Omega)$.

Finally, given the reproductive solution $(\boldsymbol{v}, \eta)$ associated to $\boldsymbol{v}(0)=\boldsymbol{v}(T):=\boldsymbol{v}_{0}$ and $\eta(0)=$ $\eta(T):=\eta_{0}$, then $(\boldsymbol{v}, \eta)$ is the (unique) solution of the initial-boundary problem associated to the initial data $\left(\boldsymbol{v}_{0}, \eta_{0}\right)$, which is defined for all time $t \in(0, \infty)$. Moreover, this solution is T-periodic, because in $(T, 2 T)$ must be equal to the reproductive solution defined as $\overline{\boldsymbol{v}}(t)=\boldsymbol{v}(t-T)$ and $\bar{\eta}(t)=\eta(t-T)$ (which verifies $\boldsymbol{v}(T)=\boldsymbol{v}(2 T)=\boldsymbol{v}_{0}$ and $\left.\eta(T)=\eta(2 T)=\eta_{0}\right)$ and so on.

Acknowledgments: The first author was partially supported by DGI-MEC (Spain), Grant MTM2009-12927.The second author was partially supported by Grant Fondecyt 1090510. The third author was partially supported by Fondecyt (Chile), Grant 1080628 and by DGI-MEC (Spain), Grant MTM2009-12927.

\section{References}

[1] R. A. Adams, Sobolev spaces. Academic Press, New York, 1975.

[2] J.L. Boldrini, M.A. Rojas-Medar, M.D. Rojas-Medar. On existence and uniqueness of stationary bioconvective flow, Submitted.

[3] J.L. Boldrini, M.A. Rojas-Medar, M.D. Rojas-Medar. On existence and unqueness of bioconvective flow, Submitted.

[4] B. Climent-Ezquerra, F. Guillén-González, M.A. Rojas-Medar, Regular time-reproductive solutions for generalized Boussinesq model with Neumannboundary conditions for temperature, Proc. R. Soc. A 463 (2007)

[5] B. Climent-Ezquerra, F. Guillén-González, M.J. Moreno-Iraberte. Regularity and Timeperiodicity for a Nematic Liquid Crystal model, Nonlinear Analysis Theory, Methods and Applications. Vol. 71. (2009) pp. 539-549.

[6] B. Climent-Ezquerra, F. Guillén-González. Global in time solution and time-periodicty for a Smectic-A Liquid Crystal Model, doi:10.3934/cpaa.2010.9. Pure and Applied Analisys, Volume 9, Number 6, November 2010. 
[7] D. Gilbarg, N.S. Trudinger, Elliptic Partial Differential Equations of Second Order. BerlinHeidelberg-New York-Tokyo, Springer-Verlag 1983.

[8] D. Joseph, Stability of Fluid Motion II. Springer, Berlin, 1976.

[9] Y. Kan-On, K. Narukawa and Y. Teramoto, On the equations of bioconvective flow, J. Math. Kyoto Univ. (JMKYAZ), Vol.32, Nro. 1, 1992, 135-153.

[10] J. Necas, Les Méthodes Directes en Téhorie des Équations Elliptiques. Paris, Masson, 1967.

[11] S. Mikhlin, The Problem of the Minimum of a Quadratic Functional, Holden Day, San Francisco, 1965.

[12] Y. Moribe, On the bioconvection of Tetrahymena pyriformis. Master's Thesis, Osaka University, 1973.

[13] C. Morrey, Multiple integrals in the calculus of variations, Springer, Berlin, 1966.

[14] O.A. Ladyzenskaja, N.N. Ural'ceva, Equations aux Dérives Partielles de type elliptique. Paris Dunod, 1968

[15] M. Levandowsky, W. S. Childress, S. H. Hunter e E. A. Spiegel, A mathematical model of pattern formation by swimming microorganisms, J. Protozoology, Vol.22, 1975, pp 296-306.

[16] F.H. Lin, C. Liu, Non-parabolic dissipative systems modelling the flow of liquid crystals, Comm. Pure Appl. Math. 4 (1995) 501-537.

[17] S. Lorca, J. L. Boldrini, The initial value problem for a generalized Boussinesq model, Nonlinear Analysis, Vol. 36, Number 4, 457-480.

[18] G. Mulone, F. Salemi, On the existence of hidrodynamic motion in a domain with free boundary type conditions, Meccanica, Vol.18, Number 3, 1983, 136-144.

[19] V. Solonnikov, V. Scadilov, On a boundary value problem for a stationary system of NavierStokes equations, Proc. Steklov Inst. Math., Vol.125, 1973, 186-199.

[20] R. Temam, Navier-Stokes equations-Theory and numerical analysis (revised edition), NorthHolland Publ. Comp., Amsterdam, 1979. 